\title{
Representation of certain homogeneous Hilbertian operator spaces and applications
}

\author{
Marius Junge* and Quanhua $\mathrm{Xu}^{\dagger}$
}

\begin{abstract}
Following Grothendieck's characterization of Hilbert spaces we consider operator spaces $F$ such that both $F$ and $F^{*}$ completely embed into the dual of a $\mathrm{C}^{*}$-algebra. Due to Haagerup/Musat's improved version of Pisier/Shlyakhtenko's Grothendieck inequality for operator spaces, these spaces are quotients of subspaces of the direct sum $C \oplus R$ of the column and row spaces (the corresponding class being denoted by $Q S(C \oplus R)$ ). We first prove a representation theorem for homogeneous $F \in Q S(C \oplus R)$ starting from the fundamental sequences

$$
\Phi_{c}(n)=\left\|\sum_{k=1}^{n} e_{k 1} \otimes e_{k}\right\|_{C \otimes_{\min } F}^{2} \quad \text { and } \quad \Phi_{r}(n)=\left\|\sum_{k=1}^{n} e_{1 k} \otimes e_{k}\right\|_{R \otimes_{\min } F}^{2}
$$

given by an orthonormal basis $\left(e_{k}\right)$ of $F$. Under a mild regularity assumption on these sequences we show that they completely determine the operator space structure of $F$ and find a canonical representation of this important class of homogeneous Hilbertian operator spaces in terms of weighted row and column spaces. This canonical representation allows us to get an explicit formula for the exactness constant of an $n$-dimensional subspace $F_{n}$ of $F$ :

$$
e x\left(F_{n}\right) \sim\left[\frac{n}{\Phi_{c}(n)} \Phi_{r}\left(\frac{\Phi_{c}(n)}{\Phi_{r}(n)}\right)+\frac{n}{\Phi_{r}(n)} \Phi_{c}\left(\frac{\Phi_{r}(n)}{\Phi_{c}(n)}\right)\right]^{1 / 2} .
$$

In the same way, the projection (=injectivity) constant of $F_{n}$ is explicitly expressed in terms of $\Phi_{c}$ and $\Phi_{r}$ too. Orlicz space techniques play a crucial role in our arguments. They also permit us to determine the completely 1-summing maps in Effros and Ruan's sense between two homogeneous spaces $E$ and $F$ in $Q S(C \oplus R)$. The resulting space $\Pi_{1}^{o}(E, F)$ isomorphically coincides with a Schatten-Orlicz class $S_{\varphi}$. Moreover, the underlying Orlicz function $\varphi$ is uniquely determined by the fundamental sequences of $E$ and $F$. In particular, applying these results to the column subspace $C_{p}$ of the Schatten $p$-class, we find the projection and exactness constants of $C_{p}^{n}$, and determine the completely 1-summing maps from $C_{p}$ to $C_{q}$ for any $1 \leq$ $p, q \leq \infty$.
\end{abstract}

\footnotetext{
* Partially supported by NSF DMS 05-56120.

$\dagger$ Partially supported by ANR 06-BLAN-0015.

2000 Mathematics subject classification: Primary 46L07; Secondary 47L25

Key words and phrases: Column and row spaces, homogeneous Hilbertian operator spaces, concrete representations, Orlicz norms, exactness, injectivity, completely 1-summing maps.
} 


\section{Introduction}

Hilbert spaces are by far the most important examples among general Banach spaces. Indeed, much research in Banach space theory relies on weakening abstract properties of Hilbert spaces and studies the class of Banach spaces satisfying these weaker properties. This applies in particular to the notions of type and cotype (see [P1]), as well as to spaces with unconditional martingale differences (so called UMD spaces) or equivalently to spaces for which the Hilbert transform continuously extends to the vector-valued setting (see [Bu1], Bu2] and [B])

In the theory of quantized Banach spaces, i.e., the theory of operator spaces, the class of Hilbert spaces is also quantized. In other words, for a given Hilbert space $F$ there exist numerous isometric embeddings of $F$ into the space $B(H)$ of bounded operators on a Hilbert space $H$, which give rise to very different matrix norms on $F$. First examples that one should have in mind are, of course, the column and row spaces

$$
C=\overline{\operatorname{span}}\left\{e_{k 1}: k \in \mathbb{N}\right\} \subset B\left(\ell_{2}\right) \quad \text { and } \quad R=\overline{\operatorname{span}}\left\{e_{1 k}: k \in \mathbb{N}\right\} \subset B\left(\ell_{2}\right) .
$$

We may think of $C$ as a Hilbert space with a column spin and $R$ with a row spin. Both $C$ and $R$ are isometric to $\ell_{2}$ as Banach spaces. They are, however, extremely different as operator spaces. The spaces $C$ and $R$ are omnipresent in operator space theory. For instance, Pisier's operator Hilbert space $O H$ can be constructed from them via complex interpolation.

The spaces $C, R$ and $O H$ are examples of homogeneous Hilbertian operator spaces. Recall that an operator space $F \subset B(H)$ is called Hilbertian, respectively, homogeneous if it is isomorphic to a Hilbert space at the Banach space level, respectively, if every bounded linear map $T$ on $F$ is automatically completely bounded. Let us point out that these spaces are dramatically different from the operator space structures on $\ell_{2}$ constructed by Oikhberg and Ricard $[\mathrm{OR}$ ] which allow only very few completely bounded maps.

The importance of $C$ and $R$ in operator space theory is also illustrated by the following noncommutative analogue of Grothendieck's abstract characterization of Hilbert spaces in the commutative theory. For a (separable) operator space $F$ both $F$ and $F^{*}$ completely embed in a noncommutative $L_{1}$-space if and only if $F$ is completely isomorphic to a quotient of a subspace of the direct sum $C \oplus R$. The "only if" part is due to Pisier/Shlyakhtenko [PS] with an exactness assumption and Haagerup/Musat HM2 in the full generality; the "if" part was proved independently by the present authors (see [J2 and $[\mathrm{X}]$ ). Let $Q S(C \oplus R)$ denote the class of quotients of subspaces of $C \oplus R$ and $H Q S(C \oplus R)$ the subclass of homogeneous spaces. This last class is the main concern of the present paper. We will study various properties of it.

In the literature we find a very particular construction of spaces in $H Q S(C \oplus R)$ closely related to quasi-free states. Let $u_{c}$ and $u_{r}$ be two weights on a measure space $(\Omega, \nu)$. Then we may consider the weighted column and row spaces $L_{2}^{c}\left(u_{c}\right)=B\left(\mathbb{C}, L_{2}\left(u_{c}\right)\right), L_{2}^{r}\left(u_{r}\right)=B\left(L_{2}\left(u_{r}\right), \mathbb{C}\right)$ and their sum

$$
L_{2}^{c}\left(u_{c}\right)+L_{2}^{r}\left(u_{r}\right)=\left\{a+b: a \in L_{2}^{c}\left(u_{c}\right), b \in L_{2}^{r}\left(u_{r}\right)\right\} .
$$

It turns out that this sum (which is no longer direct) can be realized in the predual of a quasifree von Neumann algebra (either in the free or classical sense). Clearly, $L_{2}^{c}\left(u_{c}\right)+L_{2}^{r}\left(u_{r}\right)$ is a quotient of $L_{2}^{c}\left(u_{c}\right) \oplus L_{2}^{r}\left(u_{r}\right)$. However, we may also consider the subspace of constant functions of $L_{2}^{c}\left(u_{c} ; \ell_{2}\right)+L_{2}^{r}\left(u_{r} ; \ell_{2}\right):$

$$
K_{u_{c}, u_{r}}=\left\{1 \otimes x: x \in \ell_{2}\right\} \subset L_{2}^{c}\left(u_{c} ; \ell_{2}\right)+L_{2}^{r}\left(u_{r} ; \ell_{2}\right) .
$$

Here $L_{2}\left(u_{c} ; \ell_{2}\right)=L_{2}\left(u_{c}\right) \otimes_{2} \ell_{2}$ denotes the $\ell_{2}$-valued weighted $L_{2}$-space, and the two weights are assumed to satisfy

$$
\int_{\Omega} \min \left(u_{c}, u_{r}\right) d \nu<\infty
$$

Probabilistically, we may think of $K_{u_{c}, u_{r}}$ as obtained by a family of independent copies of a single variable $v(1)$, where $v: L_{2}^{c}\left(u_{c}\right)+L_{2}^{r}\left(u_{r}\right) \rightarrow L_{1}(\mathcal{N})$ is the complete embedding using a quasifree algebra $\mathcal{N}$. Hence the infinite tensor product $V=v^{\otimes \infty}$ yields an embedding of $K_{u_{c}}, u_{r}$ in 
$L_{1}\left(\mathcal{N}^{\otimes \infty}\right)$. Note that in the classical setting $\mathcal{N}^{\otimes \infty}$ will be either the hyperfinite $\mathrm{II}_{1}$ or $\mathrm{III}_{1}$ factor. This construction is motivated by the previous works on $O H$ which admits such a description. We refer to [J1], [J2, [P6] and $[\mathrm{X}]$ for more details and related results.

The spaces of type $K_{u_{c}, u_{r}}$ clearly belong to $H Q S(C \oplus R)$. The first main result of this paper states that almost all spaces in $H Q S(C \oplus R)$ admit such representations.

Theorem 0.1 Let $F \in H Q S(C \oplus R)$ be infinite dimensional. Then there exist constants $\lambda, \gamma \geq 0$ and two weights $u_{c}, u_{r}$ on $\mathbb{R}$ with respect to Lebesgue measure such that $F$ is completely isomorphic to the intersection $\lambda C \cap \gamma R \cap K_{u_{c}}, u_{r}$. Moreover, under a mild assumption on the following functions

$$
\Phi_{c}(n)=\left\|\sum_{k=1}^{n} e_{k 1} \otimes e_{k}\right\|_{C \otimes_{\min } F}^{2} \quad \text { and } \quad \Phi_{r}(n)=\left\|\sum_{k=1}^{n} e_{1 k} \otimes e_{k}\right\|_{R \otimes_{\min } F}^{2}, \quad n \in \mathbb{N},
$$

where $\left(e_{k}\right)$ denotes an orthonormal basis of $F$, we have $\lambda=\gamma=0$ and the pair $\left(u_{c}, u_{r}\right)$ is uniquely determined, up to equivalence, by $\Phi_{c}$ and $\Phi_{r}$.

We will call the two functions in (0.1) the fundamental functions (or sequences) of $F$ and denote them by $\Phi_{c, F}$ and $\Phi_{r, F}$ if we wish to refer to $F$ explicitly. The mild assumption mentioned previously is the following: there exist positive constants $c, d$ and $\alpha, \beta$ with $0<\alpha \leq \beta<1$ such that

$$
c\left(\frac{n}{k}\right)^{\alpha} \leq \frac{\Phi_{c}(n)}{\Phi_{c}(k)} \leq d\left(\frac{n}{k}\right)^{\beta} \quad \text { and } \quad c\left(\frac{n}{k}\right)^{\alpha} \leq \frac{\Phi_{r}(n)}{\Phi_{r}(k)} \leq d\left(\frac{n}{k}\right)^{\beta} \quad, \quad \forall n \geq k \geq 1 .
$$

In this case $F$ will be called regular. We will systematically extend $\Phi_{c}$ and $\Phi_{r}$ to continuous functions on $\mathbb{R}_{+}$(still denoted by the same symbols), for instance, piecewise linearly. We can even assume, by perturbation, that $\Phi_{c}$ and $\Phi_{r}$ are increasing on $\mathbb{R}_{+}$. In particular, under the mild regularity assumption, every homogenous space in $Q S(C \oplus R)$ admits a complete embedding in the predual of the hyperfinite $\mathrm{III}_{1}$ factor.

Theorem 0.1 shows that the fundamental functions $\Phi_{c}$ and $\Phi_{r}$ completely determine the operator space structure of a regular $F \in H Q S(C \oplus R)$. We understand this theorem as a classification result of nice Hilbertian operator spaces (the class $H Q S(C \oplus R)$ ) which should play a similar role for operator spaces as Hilbert spaces do for general Banach spaces. Although much work is left to be done in this direction, we show that the fundamental functions $\Phi_{c}$ and $\Phi_{r}$ do allow us to calculate fundamental invariants of the operator space $F$. Indeed, a new feature in operator space theory is the notion of exactness. An operator space $F$ is called exact if

$$
e x(F)=\sup _{E \subset F, \operatorname{dim}} \inf _{E \subset \infty} d_{G \subset \mathbb{K}\left(\ell_{2}\right)}(E, G)<\infty,
$$

where $\mathbb{K}\left(\ell_{2}\right)$ denotes the space of compact operators on $\ell_{2}$. This notion was first investigated by Kirchberg [K2], [K3] and [K1] in the category of $\mathrm{C}^{*}$-algebras and then by Pisier [P2] for operator spaces. In fact, a $C^{*}$-algebra $A$ is exact in the categorial sense if and only if $\operatorname{ex}(A)=1$. Thus knowing that $e x(F)>1$ implies that no $C^{*}$-algebra generated by some copies of $F$ can be exact. For example, the $n$-dimensional operator Hilbert space $O H_{n}$ satisfies $\operatorname{ex}\left(O H_{n}\right) \sim n^{1 / 4}$, whereas $e x(R)=1=e x(C)$.

Theorem 0.2 Let $F \in H Q S(C \oplus R)$ be regular and $F_{n}$ an n-dimensional subspace of $F$. Let $\Phi_{c}$ and $\Phi_{r}$ be the fundamental functions of $F$. Then

$$
e x\left(F_{n}\right) \sim\left[\frac{n}{\Phi_{c}(n)} \Phi_{r}\left(\frac{\Phi_{c}(n)}{\Phi_{r}(n)}\right)+\frac{n}{\Phi_{r}(n)} \Phi_{c}\left(\frac{\Phi_{r}(n)}{\Phi_{c}(n)}\right)\right]^{1 / 2},
$$

where the equivalence constants depend only on the homogeneity constant of $F$ and the constants in the regularity condition (0.2). 
Our next result concerns the projection constants of the spaces $F_{n}$. Calculating projection constants for classical Banach spaces has advanced the general knowledge and theory, in particular the local theory. We think that this particular class of operator spaces should serve as a testing class for understanding general features of operator spaces, notably because this class is amenable to concrete calculations. Recall that the projection or injectivity constant of $F$ is defined by

$$
\lambda_{c b}(F)=\inf \left\{\|P\|_{c b}: F \subset B(H) \text { as subspace, } P: B(H) \rightarrow F \text { projection }\right\} .
$$

Theorem 0.3 Under the same assumption of Theorem 0.2 we have

$$
\begin{aligned}
\frac{1}{\lambda_{c b}\left(F_{n}\right)} \sim & {\left[\frac{1}{\Phi_{c, F}(n) \Phi_{r, F^{*}}(n)}+\frac{1}{\Phi_{r, F}(n) \Phi_{c, F^{*}}(n)}\right]^{1 / 2}+} \\
\frac{1}{\sqrt{n}} & {\left[\int_{1}^{\Phi_{c, F^{*}}(n)} \frac{\Phi_{r, F}\left(\Phi_{c, F^{*}}^{-1}(t)\right)}{\Phi_{c, F^{*}}^{-1}(t)} d t+\int_{1}^{\Phi_{r, F^{*}}(n)} \frac{\Phi_{c, F}\left(\Phi_{r, F^{*}}^{-1}(t)\right)}{\Phi_{r, F^{*}}^{-1}(t)} d t+\right.} \\
& \left.\int_{1}^{\Phi_{c, F}(n)} \frac{\Phi_{r, F^{*}}\left(\Phi_{c, F}^{-1}(t)\right)}{\Phi_{c, F}^{-1}(t)} d t+\int_{1}^{\Phi_{r, F}(n)} \frac{\Phi_{c, F^{*}}\left(\Phi_{r, F}^{-1}(t)\right)}{\Phi_{r, F}^{-1}(t)} d t\right]^{1 / 2} .
\end{aligned}
$$

Here $\Phi^{-1}$ denotes the generalized inverse of a nondecreasing function $\Phi$ on $\mathbb{R}_{+}$.

It is interesting to note that the right hand side above is symmetric in $F$ and $F^{*}$. Consequently, for a regular $F \in H Q S(C \oplus R)$ we have $\lambda_{c b}\left(F_{n}\right) \sim \lambda_{c b}\left(F_{n}^{*}\right)$ (see Proposition 5.1 for a more general result of this kind). We will show in Theorem 3.11 below that if $F \in H Q S(C \oplus R)$ is regular, so is $F^{*}$ and their fundamental functions are linked as follows

$$
\Phi_{c, F}(n) \Phi_{c, F^{*}}(n) \sim n \quad \text { and } \quad \Phi_{r, F}(n) \Phi_{r, F^{*}}(n) \sim n .
$$

Let $C_{p}$ denote the column $p$-space, which is the column subspace of the Schatten $p$-class. It is known that $C_{p} \in Q S(C \oplus R)$ (see [J1] and $[\mathrm{X}]$ ). It is also easy to calculate its fundamental functions

$$
\Phi_{c, C_{p}}(t)=t^{1 / p^{\prime}} \quad \text { and } \quad \Phi_{r, C_{p}}(t)=t^{1 / p}, \quad t \in \mathbb{R}_{+},
$$

where $p^{\prime}$ denotes the conjugate index of $p$. Thus $C_{p}$ is regular for $1<p<\infty$, and by the preceding theorems, we immediately find

$$
e x\left(C_{p}^{n}\right) \sim n^{1 / p p^{\prime}}
$$

and

$$
\lambda_{c b}\left(C_{p}^{n}\right) \sim n^{1 / \max \left(p, p^{\prime}\right)} \text { if } p \neq 2 \quad \text { and } \quad \lambda_{c b}\left(C_{2}^{n}\right) \sim \frac{\sqrt{n}}{\sqrt{\log (n+1)}} .
$$

The estimates above for $p \neq 2$ are new. For $p=2$ the estimate on $\operatorname{ex}\left(C_{2}^{n}\right)$ is due to Pisier ([P2]; see also [P5, Theorem 21.5]) and that on $\lambda_{c b}\left(C_{2}^{n}\right)$ is the combination of [PS, Corollary 3.7] and [J1, Corollary 4.11].

One main new feature in our arguments is the use of Orlicz space techniques. This is the first time that these techniques are employed in operator space theory. They also allow us to describe the completely 1-summing maps in Effros-Ruan's sense between two spaces $E$ and $F$ in $H Q S(C \oplus R)$. Let $\Pi_{1}^{o}(E, F)$ denote the space of all completely 1-summing maps from $E$ to $F$, equipped with the completely 1 -summing norm $\pi_{1}^{o}$. Using the representation Theorem 0.1 we show that $\Pi_{1}^{o}(E, F)$ isomorphically coincides with a Schatten-Orlicz class $S_{\varphi}$ (see the beginning of section 4 for the definition of $S_{\varphi}$ ). Since $S_{\varphi}$ is determined, up to an equivalent norm, by the fundamental sequence of the underlying Orlicz function $\varphi$, this result reduces the determination of $\Pi_{1}^{o}(E, F)$ to that of the sequence $\left(\pi_{1}^{o}\left(\mathrm{id}_{n}\right)\right)_{n \geq 1}$, where $\mathrm{id}_{n}$ is the formal identity from an $n$-dimensional subspace of $E$ to another one of $F$. Choose two orthonormal bases $\left(e_{k}\right)$ and $\left(f_{k}\right)$ of $E$ and $F$, respectively. Then 
$\operatorname{id}_{n}$ is the map such that $\operatorname{id}_{n}\left(e_{k}\right)=f_{k}$ for $k \leq n$ and $\operatorname{id}_{n}\left(e_{k}\right)=0$ for $k>n$. The homogeneity of $E$ and $F$ shows that $\pi_{1}^{o}\left(\mathrm{id}_{n}\right)$ is independent of particular choice of $\left(e_{k}\right)$ and $\left(f_{k}\right)$.

Only with the help of this reduction result we can determine the whole space $\Pi_{1}^{o}(E, F)$. In many situations it is hard or even impossible to estimate $\pi_{1}^{o}(u)$ for any $u: E \rightarrow F$ but it is relatively easier to determine $\pi_{1}^{o}\left(\mathrm{id}_{n}\right)$ via concrete integral calculations. This is indeed the case for $E=F=O H$. Then $\pi_{1}^{o}\left(\operatorname{id}_{n}: O H_{n} \rightarrow O H_{n}\right)$ was determined by the first named author in [J1]: $\pi_{1}^{o}\left(\mathrm{id}_{n}: O H_{n} \rightarrow O H_{n}\right) \sim \sqrt{n \log (n+1)}$ uniformly in $n \in \mathbb{N}$. Note that this estimate is equivalent to that of $\gamma_{c b}\left(O H_{n}\right)$ in Theorem 0.3 . Thus the previous reduction result implies that $\Pi_{1}^{o}(O H)=S_{\psi}$, where $S_{\psi}$ is the Schatten-Orlicz class associated to the Orlicz function $\psi$ defined by $\psi(t)=t^{2} \log (t+1 / t)$. This result is in strong contrast with the corresponding result in Banach space theory. Recall that a map $u: X \rightarrow Y$ between two Banach spaces is 1-summing if there exists a constant $\lambda>0$ such that for all finite sequences $\left(x_{k}\right) \subset X$

$$
\sum_{k}\left\|u\left(x_{k}\right)\right\| \leq \lambda \sup \left\{\sum_{k}\left|\xi\left(x_{k}\right)\right|:\|\xi\| \leq 1, \xi \in X^{*}\right\} .
$$

It is well known that a map $u$ on a Hilbert space $H$ is 1 -summing if and only if $u$ is a Hilbert-Schmidt operator (see, for instance, [P1]).

If both $E$ and $F$ are regular, we can do much better. In this case, we have an explicit formula for $\pi_{1}^{o}\left(\mathrm{id}_{n}\right)$ in terms of the fundamental functions of $E$ and $F$.

Theorem 0.4 Let $E, F \in H Q S(C \oplus R)$ be regular. Then $\Pi_{1}^{o}(E, F)=S_{\varphi}$ for some Orlicz function $\varphi$ whose fundamental sequence $\left(\varphi_{n}\right)_{n \geq 1}$ is given by

$$
\begin{aligned}
\varphi_{n}^{2} \sim & \Phi_{c, E^{*}}(n) \Phi_{r, F}(n)+\Phi_{r, E^{*}}(n) \Phi_{c, F}(n)+ \\
n & {\left[\int_{1}^{\Phi_{c, E^{*}}(n)} \frac{\Phi_{r, F}\left(\Phi_{c, E^{*}}^{-1}(t)\right)}{\Phi_{c, E^{*}}^{-1}(t)} d t+\int_{1}^{\Phi_{r, E^{*}}(n)} \frac{\Phi_{c, F}\left(\Phi_{r, E^{*}}^{-1}(t)\right)}{\Phi_{r, E^{*}}^{-1}(t)} d t+\right.} \\
& \left.\int_{1}^{\Phi_{c, F}(n)} \frac{\Phi_{r, E^{*}}\left(\Phi_{c, F}^{-1}(t)\right)}{\Phi_{c, F}^{-1}(t)} d t+\int_{1}^{\Phi_{r, F}(n)} \frac{\Phi_{c, E^{*}}\left(\Phi_{r, F}^{-1}(t)\right)}{\Phi_{r, F}^{-1}(t)} d t\right],
\end{aligned}
$$

where the equivalence constants depend only on the homogeneity and regularity constants of $E$ and F.

Applying this theorem to the column $p$-spaces, we immediately determine the whole space $\Pi_{1}^{o}\left(C_{p}, C_{q}\right)$. In particular, we recover the result on $\pi_{1}^{o}\left(\mathrm{id}_{n}: C_{2}^{n} \rightarrow C_{2}^{n}\right)$ of [J1] quoted above by simpler arguments which avoid some tedious integral calculations (for instance, we do not use the duality argument of [J1]).

The paper is organized as follows. After a preliminary section on operator space theory, we prove in section 2 the first part of Theorem 0.1, which is reformulated as Theorem 2.3. The uniqueness part of Theorem 0.1 is proved in section 3. There the Orlicz space techniques mentioned previously appear for the first time (see the proof of Lemma 3.4). Section 4 deals with completely 1-summing maps. The first main result there states that for $E$ and $F$ in $H Q S(C \oplus R)$ we have $\Pi_{1}^{o}(E, F)=S_{\varphi}$ isomorphically for some Orlicz function $\varphi$. Another main result is Theorem 0.4. Section 5 concerns the projection constants and exactness constants. There Theorems 0.3 and 0.2 are proved. In the last section we apply all these results to the column spaces $C_{p}$ and their sums and intersections with the row spaces $R_{p}$. Consequently, we determine all previous objects for these spaces.

The techniques developed in this paper allow to obtain natural operator space structures on certain Schatten-Orlicz spaces. They also permit to deal with completely $p$-summing maps in Pisier's sense. These subjects will be pursued elsewhere. We refer to Yew's paper $[\mathrm{Y}]$ for the study of completely $p$-summing maps on $O H$.

All spaces considered in this paper will be separable and infinite dimensional, unless explicitly stated otherwise. The letter $c$ will often denote a universal constant. We will frequently use the notation $A \sim_{c} B$ to abbreviate the two-sided inequality $c^{-1} B \leq A \leq c B$. 


\section{Preliminaries}

In this section we collect some preliminaries necessary to the whole paper. We will use standard notions and notation from operator space theory. Our references are ER2] and [P5]. An operator space $E$ is called homogeneous if there exists a constant $\lambda$ such that every bounded map $u$ on $E$ is completely bounded and $\|u\|_{c b} \leq \lambda\|u\|$. In this case we also say that $E$ is $\lambda$-homogeneous. $E$ is called Hilbertian if $E$ is isomorphic to a Hilbert space (at the Banach space level). If we wish to emphasize the isomorphism constant $\lambda$ between $E$ and the Hilbert space, we say that $E$ is $\lambda$-Hilbertian.

The Schatten classes $S_{p}$ will be frequently used in this paper. Recall that $S_{1}$ is the trace class, $S_{2}$ the Hilbert-Schmidt class and $S_{\infty}=B\left(\ell_{2}\right)$. These spaces are equipped with their natural operator space strictures as introduced in [P4]. We will also need their vector-valued versions. Let $E$ be an operator space. Define $S_{\infty}[E]=S_{\infty} \otimes_{\min } E$ and $S_{1}[E]=S_{1} \widehat{\otimes} E$. Here $\otimes_{\min }$ and $\widehat{\otimes}$ denote, respectively, the minimal (injective) and projective tensor products in the category of operator spaces. For $1<p<\infty$ the space $S_{p}[E]$ is defined as the complex interpolation space $\left(S_{\infty}[E], S_{1}[E]\right)_{1 / p}$. Only $S_{\infty}[E]$ and $S_{1}[E]$ will be needed later. We refer to $[\mathrm{P} 4$ for more information.

We now recall the direct sum of two operator spaces $E$ and $F$. Let $1 \leq p \leq \infty . E \oplus_{p} F$ denotes the direct sum of $E$ and $F$ in the $\ell_{p}$-sense (see [P4]). Note that $E \oplus_{p} F$ is completely isomorphic to $E \oplus_{q} F$ for any $1 \leq q \leq \infty$ with universal constants. This allows us to drop the subscript $p$ from $E \oplus_{p} F$, a convention adopted throughout the paper. Note that if both $E$ and $F$ are Hilbertian, so is $E \oplus F$. However, in the 1-Hilbertian case, only $E \oplus_{2} F$ is 1-Hilbertian.

We will need to consider the sum and intersection of a compatible couple $(E, F)$ of operator spaces. The intersection $E \cap F$ is the diagonal subspace of $E \oplus F$ and the sum $E+F$ is

$$
E+F=\{a+b: a \in E, b \in F\} .
$$

Note that $E+F$ is the quotient of $E \oplus F$ by the subspace $\{(a, b): a+b=0\}$.

The column and row spaces, $C$ and $R$, are the two major objects of this paper. Recall that $C$ and $R$ are respectively the (first) column and row subspaces of $S_{\infty}$. More precisely, $C$ is the subspace of $S_{\infty}$ consisting of matrices whose all entries but those in the first column vanish. As Banach spaces both $C$ and $R$ are isometric to $\ell_{2}$, so they are 1-Hilbertian. This allows us to identify both $C$ and $R$ with $\ell_{2}$ at the Banach space level. Accordingly, we will often identify the canonical bases $\left(e_{k 1}\right)$ of $C$ and $\left(e_{1 k}\right)$ of $R$ with $\left(e_{k}\right)$ of $\ell_{2}$. It is easy to see that the operator space structures of $C$ and $R$ are determined as follows. Let $\left(x_{k}\right)$ be a finite sequence in $S_{\infty}$. Then

$$
\left\|\sum_{k} x_{k} \otimes e_{k}\right\|_{S_{\infty}[C]}=\left\|\sum_{k} x_{k}^{*} x_{k}\right\|_{\infty}^{1 / 2}, \quad\left\|\sum_{k} x_{k} \otimes e_{k}\right\|_{S_{\infty}[R]}=\left\|\sum_{k} x_{k} x_{k}^{*}\right\|_{\infty}^{1 / 2} .
$$

This implies that $C$ and $R$ are 1-homogeneous. More generally, if $H$ is a Hilbert space, the column and row spaces based on $H$ are $H^{c}=B(\mathbb{C}, H)$ and $H^{r}=B(H, \mathbb{C})$, respectively. If $H$ is separable and infinite dimensional, we recover $C$ and $R$. On the other hand, if $\operatorname{dim} H=n<\infty$, we get $C^{n}$ and $R^{n}$, the $n$-dimensional versions of $C$ and $R$.

We will be interested only in the homogeneous spaces in $Q S(C \oplus R)$. Here given an operator space $E$ we use $Q S(E)$ to denote the family of all quotients of subspaces of $E$. This coincides with the family of all subspaces of quotients of $E$. The subfamily of homogeneous spaces of $Q S(C \oplus R)$ is denoted by $H Q S(C \oplus R)$. We will study several properties of these spaces. It is well-known that Pisier's operator Hilbert space $O H$ constructed in [P3] belongs to $H Q S(C \oplus R)$ (see [P5, Exercice 7.9] and also [P6]). More generally, the column $p$-space $C_{p}$ belongs to $H Q S(C \oplus R)$ too (see [J1] and $[\mathrm{X}]$ ).

Recall that $C_{p}$ and $R_{p}$ denote the (first) column and row subspaces of $S_{p}$. Their $n$-dimensional versions are denoted by $C_{p}^{n}$ and $R_{p}^{n}$, respectively. Note that $C_{\infty}$ and $R_{\infty}$ are just $C$ and $R$. On the other hand, $C_{2}$ and $R_{2}$ coincide completely isometrically with $O H$. Like $C$ and $R, C_{p}$ and $R_{p}$ 
are also 1-homogenous and 1-Hilbertian. We will also identify $C_{p}$ and $R_{p}$ with $\ell_{2}$ as Banach spaces and use $\left(e_{k}\right)$ to denote their common canonical basis. We have the following completely isometric identities: for any $1 \leq p \leq \infty$

$$
\left(C_{p}\right)^{*} \cong C_{p^{\prime}} \cong R_{p} \text { and }\left(R_{p}\right)^{*} \cong R_{p^{\prime}} \cong C_{p},
$$

where $p^{\prime}$ denotes the index conjugate to $p . C_{p}$ and $R_{p}$ can be also defined via interpolation from $C$ and $R$. We view $(C, R)$ as a compatible couple by identifying both of them with $\ell_{2}$ (at the Banach space level). Then

$$
C_{p}=(C, R)_{1 / p}=\left(C_{\infty}, C_{1}\right)_{1 / p} \quad \text { and } \quad R_{p}=(R, C)_{1 / p}=\left(R_{\infty}, R_{1}\right)_{1 / p} .
$$

We refer to $\mathrm{P} 3$ ] and $[\mathrm{P} 4$ for all these elementary facts.

If $E$ is an operator space, $C_{p}[E]$ (resp. $R_{p}[E]$ ) denotes the closure of $C_{p} \otimes E$ (resp. $R_{p} \otimes E$ ) in $S_{p}[E]$. Thus $C[E]=C \otimes_{\min } E$ and $C_{1}[E]=C_{1} \widehat{\otimes} E$.

We end this preliminary section by introducing the notion of completely 1 -summing maps. Let $x: E \rightarrow F$ be a map between two operator spaces. $x$ is called completely 1-summing if the map $\operatorname{id} \otimes x$ is bounded from $S_{1} \otimes_{\min } E$ to $S_{1}[F]$. In this case we define $\pi_{1}^{o}(x)$ to be the norm of id $\otimes x$ and call it the completely 1-summing norm of $x$. The space of all completely 1-summing maps from $E$ to $F$ is denoted by $\Pi_{1}^{o}(E, F)$ and equipped with the completely 1-summing norm. This is a Banach space. It is easy to check that $\Pi_{1}^{o}(E, F)$ is an ideal in the following sense. Let $E_{1}$ and $F_{1}$ be two other operator spaces and let $y \in C B\left(E_{1}, E\right)$ and $z \in C B\left(F, F_{1}\right)$. Then $z x y \in \Pi_{1}^{o}\left(E_{1}, F_{1}\right)$ for any $x \in \Pi_{1}^{o}(E, F)$ and $\pi_{1}^{o}(z x y) \leq\|z\|_{c b} \pi_{1}^{o}(x)\|y\|_{c b}$. We refer to [ER1] and [P4] for more information.

\section{Representations of homogeneous spaces in $Q S(C \oplus R)$}

In this section we consider spaces in $H Q S(C \oplus R)$. The main result is a representation theorem for these spaces (the first part of Theorem 0.1 . . Let $(\Omega, \nu)$ be a measure space and $u$ a weight on $\Omega$ (i.e., a nonnegative measurable function). We denote by $L_{2}(\Omega, u)$ the corresponding weighted $L_{2}$-space whose norm is given by

$$
\|f\|_{L_{2}(\Omega, u)}=\left(\int_{\Omega}|f|^{2} u d \nu\right)^{1 / 2} .
$$

Similarly, given a Banach space $X$ we define the space $L_{2}(\Omega, u ; X)$ of functions on $\Omega$ with values in $X$; the norm of $L_{2}(\Omega, u ; X)$ is defined as above by replacing the absolute value by the norm of $X$. We will need, however, only the case $X=\ell_{2}$. Then $L_{2}\left(\Omega, u ; \ell_{2}\right)$ is again a Hilbert space. $L_{2}(\Omega, u)$ and $L_{2}(\Omega, u ; X)$ will be denoted simply by $L_{2}(u)$ and $L_{2}(u ; X)$, respectively, if no confusion can occur. We will denote by $L_{2}^{c}\left(u ; \ell_{2}\right)$ (resp. $\left.L_{2}^{r}\left(u ; \ell_{2}\right)\right)$ the column (resp. row) operator space based on $L_{2}\left(u ; \ell_{2}\right)$.

Let $\left(u_{c}, u_{r}\right)$ be a pair of weights on $\Omega$ such that

$$
\int_{\Omega} \min \left(u_{c}, u_{r}\right) d \nu<\infty
$$

We will call this the weight condition and will always assume it whenever a pair of weights is considered. We view $\left(L_{2}^{c}\left(u_{c} ; \ell_{2}\right), L_{2}^{r}\left(u_{r} ; \ell_{2}\right)\right)$ as a compatible pair in the natural way. Then define

$$
G_{u_{c}, u_{r}}=L_{2}^{c}\left(u_{c} ; \ell_{2}\right)+L_{2}^{r}\left(u_{r} ; \ell_{2}\right) .
$$

This is the quotient of $L_{2}^{c}\left(u_{c} ; \ell_{2}\right) \oplus L_{2}^{r}\left(u_{r} ; \ell_{2}\right)$ by the subspace of all $\ell_{2}$-valued functions $(a, b)$ such that $a+b=0$ a.e. on $\Omega$; so $G_{u_{c}, u_{r}}$ can be viewed as a quotient of $C \oplus R$. Let $K_{u_{c}, u_{r}}$ be the subspace of constant functions of $G_{u_{c}}, u_{r}$. Identifying constant functions with vectors of $\ell_{2}$, we easily check that $K_{u_{c}, u_{r}}$ coincides (isomorphically) with $\ell_{2}$ as Banach spaces. Moreover, the isomorphism 
becomes an isometry if the underlying sum is in the $\ell_{2}$-sense. Indeed, given $x=\left(x_{k}\right) \in K_{u_{c}}, u_{r}$ we have

$$
\begin{aligned}
\|x\|_{K_{u_{c}, u_{r}}}^{2} & =\inf \left\{\sum_{k} \int_{\Omega}\left(\left|a_{k}\right|^{2} u_{c} d \nu+\left|b_{k}\right|^{2} u_{r}\right) d \nu: x_{k}=a_{k}(\omega)+b_{k}(\omega) \text { a.e. }\right\} \\
& =\sum_{k} \int_{\Omega} \inf _{0 \leq t \leq 1}\left(t^{2} u_{c}+(1-t)^{2} u_{r}\right)\left|x_{k}\right|^{2} d \nu=\int_{\Omega} \frac{u_{c} u_{r}}{u_{c}+u_{r}} \sum_{k}\left|x_{k}\right|^{2} .
\end{aligned}
$$

The last integral is finite thanks to (2.1). In the sequel we will often identify $K_{u_{c}, u_{r}}$ and $\ell_{2}$ at the Banach level and use $\left(e_{k}\right)$ to denote the canonical basis of $K_{u_{c}}, u_{r}$ too.

The operator space structure of $K_{u_{c}, u_{r}}$ is given as follows. For any finite sequence $\left(x_{k}\right) \subset S_{\infty}$

$$
\left\|\sum_{k} x_{k} \otimes e_{k}\right\|_{S_{\infty}\left[K_{\left.u_{c}, u_{r}\right]}\right.}=\inf \left\{\left\|\sum_{k} \int_{\Omega} a_{k}^{*} a_{k} u_{c} d \nu\right\|_{S_{\infty}}^{1 / 2}+\left\|\sum_{k} \int_{\Omega} b_{k} b_{k}^{*} u_{r} d \nu\right\|_{S_{\infty}}^{1 / 2}\right\}
$$

where the infimum runs over all decompositions $x_{k}=a_{k}(\omega)+b_{k}(\omega)$ a.e. on $\Omega$ with $a_{k} \in S_{\infty}\left[L_{2}^{c}\left(u_{c}\right)\right]$ and $b_{k} \in S_{\infty}\left[L_{2}^{r}(\mu)\right]$. We will show that these spaces $K_{u_{c}, u_{r}}$ are the nontrivial building blocks of spaces in $H Q S(C \oplus R)$.

Remark 2.1 If $u_{c}$ or $u_{r}$ vanishes on a subset $A \subset \Omega$, then $A$ does not contribute to $K_{u_{c}, u_{r}}$. Namely, the space $K_{u_{c}, u_{r}}$ constructed over $\Omega$ is the same as that over $\Omega \backslash A$. Thus all weights in the sequel will be assumed strictly positive unless explicitly stated otherwise.

It is sometimes convenient to work with the discrete analogue of $K_{u_{c}}, u_{r}$, i.e., when $(\Omega, \nu)$ is a discrete measure space. Consider, for instance, the case where $\Omega=\mathbb{N}$ and $\nu$ is the counting measure. Then the two weights $u_{c}$ and $u_{r}$ become two positive sequences $\left(u_{c}(j)\right)_{j \geq 1}$ and $\left(u_{r}(j)\right)_{j \geq 1}$ satisfying the following weight condition

$$
\sum_{j} \min \left(u_{c}(j), u_{r}(j)\right)<\infty .
$$

The space $G_{u_{c}, u_{r}}$ is now given by

$$
G_{u_{c}, u_{r}}=\ell_{2}^{c}\left(u_{c} ; \ell_{2}\right)+\ell_{2}^{r}\left(u_{r} ; \ell_{2}\right) .
$$

$K_{u_{c}, u_{r}}$ is the subspace of $G_{u_{c}, u_{r}}$ consisting of constant sequences.

By standard arguments it is easy to transfer the continuous case to the discrete one and vice versa. More precisely, we have the following

Proposition 2.2 (i) Let $\left(u_{c}, u_{r}\right)$ be a pair of weights on a measure space $(\Omega, \nu)$ verifying (2.1). Let $\left(d_{j}\right)$ be a positive sequence and $\left(A_{j}\right)$ a partition of $\Omega$ such that

$$
d_{j} \leq \frac{u_{r}}{u_{c}} \leq c d_{j} \quad \text { on } \quad A_{j}
$$

for some positive constant $c$. Let $\widetilde{u}_{c}=\left(\widetilde{u}_{c}(j)\right)$ and $\widetilde{u}_{r}=\left(\widetilde{u}_{r}(j)\right)$ be two sequences defined by

$$
\widetilde{u}_{c}(j)=\int_{A_{j}} u_{c} d \nu \quad \text { and } \quad \widetilde{u}_{r}(j)=d_{j} \widetilde{u}_{c}(j) .
$$

Then $\left(\widetilde{u}_{c}, \widetilde{u}_{r}\right)$ satisfies (2.3) and $K_{u_{c}, u_{r}}$ is completely isomorphic to $K_{\widetilde{u}_{c}, \widetilde{u}_{r}}$.

(ii) Conversely, given two positive sequences $\widetilde{u}_{c}$ and $\widetilde{u}_{r}$ verifying (2.3) define two weights $u_{c}$ and $u_{r}$ on $\mathbb{R}_{+}$by

$$
u_{c}=\sum_{j \geq 1} \widetilde{u}_{c}(j) \mathbb{1}_{(j-1, j]} \quad \text { and } \quad u_{r}=\sum_{j \geq 1} \widetilde{u}_{r}(j) \mathbb{1}_{(j-1, j]} .
$$

Then $u_{c}$ and $u_{r}$ satisfy (2.1) and $K_{\widetilde{u}_{c}, \widetilde{u}_{r}}$ is completely isomorphic to $K_{u_{c}}, u_{r}$, where $\mathbb{R}_{+}$is equipped with Lebesgue measure. 
Proof. (i) We have

$$
\begin{aligned}
\sum_{j} \min \left(\widetilde{u}_{c}(j), \widetilde{u}_{r}(j)\right) & =\sum_{j} \int_{A_{j}} \min \left(1, d_{j}\right) u_{c} d \nu \\
& \leq \sum_{j} \int_{A_{j}} \min \left(u_{c}, u_{r}\right) d \nu=\int_{\Omega} \min \left(u_{c}, u_{r}\right) d \nu<\infty
\end{aligned}
$$

Define a map $T$ by

$$
T(f)=\left(\frac{1}{\widetilde{u}_{c}(j)} \int_{A_{j}} f u_{c} d \nu\right)_{j \geq 1} .
$$

Then it is easy to check that $T$ is a contraction from $L_{2}\left(u_{c}\right)$ to $\ell_{2}\left(\widetilde{u}_{c}\right)$ as well as from $L_{2}\left(u_{r}\right)$ to $\ell_{2}\left(\widetilde{u}_{r}\right)$. It follows, by homogeneity, that $T \otimes$ id is completely contractive from $L_{2}^{c}\left(u_{c} ; \ell_{2}\right)$ to $\ell_{2}^{c}\left(\widetilde{u}_{c} ; \ell_{2}\right)$ and from $L_{2}^{r}\left(u_{r} ; \ell_{2}\right)$ to $\ell_{2}^{r}\left(\widetilde{u}_{r} ; \ell_{2}\right)$. Now let $\left(x_{k}\right)$ be a finite sequence in $S_{\infty}$ and consider a decomposition $x_{k}=a_{k}(\omega)+b_{k}(\omega)$ a.e. on $\Omega$. Then $x_{k}=\widetilde{a}_{j k}+\widetilde{b}_{j k}$ for all $j$, where $\widetilde{a}_{k}=T\left(a_{k}\right)$ and $\widetilde{b}_{k}=T\left(b_{k}\right)$. Moreover,

$$
\left\|\sum_{j, k} \widetilde{u}_{c}(j) \widetilde{a}_{j k}^{*} \widetilde{a}_{j k}\right\| \leq\left\|\sum_{k} \int_{\Omega} a_{k}^{*} a_{k} u_{c} d \nu\right\|, \quad\left\|\sum_{j, k} \widetilde{u}_{r}(j) \widetilde{b}_{j k} \widetilde{b}_{j k}^{*}\right\| \leq\left\|\sum_{k} \int_{\Omega} b_{k} b_{k}^{*} u_{r} d \nu\right\| .
$$

We thus deduce

$$
\left\|\sum_{k} x_{k} \otimes e_{k}\right\|_{S_{\infty}\left[K_{\tilde{u}_{c}, \tilde{u}_{r}}\right]} \leq\left\|\sum_{k} x_{k} \otimes e_{k}\right\|_{S_{\infty}\left[K_{u_{c}, u_{r}}\right]} .
$$

The converse inequality is proved similarly by using the map $T^{\prime}$ defined by

$$
T^{\prime}(x)=\sum_{j} x_{j} \mathbb{1}_{A_{j}} .
$$

Indeed, $T^{\prime}$ is contractive from $\ell_{2}\left(\widetilde{u}_{c}\right)$ to $L_{2}\left(u_{c}\right)$ and bounded from $\ell_{2}\left(\widetilde{u}_{r}\right)$ to $L_{2}\left(u_{r}\right)$ with norm $\leq \sqrt{c}$. Then as above, we deduce the missing converse inequality. Therefore, $K_{u_{c}, u_{r}}$ is completely isomorphic to $K_{\widetilde{u}_{c}, \widetilde{u}_{r}}$. This shows (i). The proof of (ii) is similar and thus omitted.

The following theorem shows that except $C, R$ and $C \cap R$ all homogeneous spaces in $Q S(C \oplus R)$ are of the form $K_{u_{c}, u_{r}}$ for some sequences $u_{c}$ and $u_{r}$. Recall that if $E$ is an operator space and $\lambda$ a positive constant, $\lambda E$ denotes the same operator space as $E$ but with norm equal to $\lambda$ times that of $E$. For convenience we also set $\lambda E=\{0\}$ if $\lambda=0$. In the latter case the intersection $\lambda C \cap \gamma R \cap K_{u_{c}, u_{r}}$ below is understood as $\gamma R \cap K_{u_{c}, u_{r}}$.

Theorem 2.3 Let $F$ be an infinite dimensional space in $H Q S(C \oplus R)$. Then there exist two constants $\lambda, \gamma \in[0,1]$ and two positive sequences $u_{c}=\left(u_{c}(j)\right)_{j \geq 1}, u_{r}=\left(u_{r}(j)\right)_{j \geq 1}$ such that $u_{c}$ and $u_{r}$ satisfy (2.3) and such that $F$ is completely isomorphic to $\lambda C \cap \gamma R \cap K_{u_{c}, u_{r}}$. Moreover, the relevant constant depends only on the homogeneity constant of $F$.

We start the proof of the theorem by some preparations. Let $S \subset C \oplus R$ be a closed subspace such that $F$ is a subspace of the quotient $(C \oplus R) / S$. By the decomposition theorem of $[\mathrm{X}]$, we find four subspaces $H_{j} \subset \ell_{2}, 0 \leq j \leq 3$ and an injective closed densely defined operator $T: H_{2} \rightarrow H_{3}$ of dense range such that

$$
S=H_{0}^{c} \oplus H_{1}^{r} \oplus \Gamma(T),
$$

where $\Gamma(T)=\{(x, T x): x \in \operatorname{Dom}(T)\}$ is the graph of $T$, viewed as a subspace of $C \oplus R$. Assume that all direct sums are in the $\ell_{2}$-sense. Then the previous decomposition of $S$ is orthogonal. This implies $H_{0} \perp H_{2}$ and $H_{1} \perp H_{3}$. On the other hand, writing the polar decomposition of $T$ and using homogeneity, we can assume that $H_{2}=H_{3}$ and $T$ is a positive operator on $H_{2}$. Next, using the spectral decomposition of $T$ and by approximation, we can further assume that $T$ has only 
point spectrum, i.e., $\mathrm{H}_{2}$ has an orthonormal basis consisting of eigenvectors of $T$. Finally, by homogeneity once more, we may fix an orthonormal basis in each $H_{j}$ which consists of vectors in the canonical basis $\left(e_{k}\right)$ of $\ell_{2}$. Moreover, the basis of $H_{2}$ is formed of eigenvectors of $T$. This choice of $S$ will be fixed in the sequel. It is to ensure the invariance of $S$ by any diagonal operator on $\ell_{2}$.

Fix an orthonormal basis $\left(f_{k}\right)$ of $F$. Then $\left(f_{k}\right)$ is completely symmetric in the following sense: there exists a constant $\lambda$ (majorized by the homogeneity constant of $F$ ) such that

$$
\left\|\sum_{k} \varepsilon_{k} a_{\pi(k)} \otimes f_{k}\right\| \leq \lambda\left\|\sum_{k} a_{k} \otimes f_{k}\right\|
$$

holds for all finite sequences $\left(a_{k}\right) \subset S_{\infty}, \varepsilon_{k}= \pm 1$ and permutations $\pi$ on $\mathbb{N}$. This is equivalent to say that $\left(f_{k}\right)$ is completely equivalent to $\left(f_{\pi(k)}\right)$ for any permutation $\pi$ of $\mathbb{N}$. By the complete equivalence of two bases $\left(f_{k}\right)$ of $F$ and $\left(g_{k}\right)$ of $G$ we mean that the map $f_{k} \mapsto g_{k}$ extends to a complete isomorphism from $F$ onto $G$. We will assume, for simplicity, that $F$ is 1-homogeneous. Set $C\left(\mathbb{N}^{2}\right)=\ell_{2}\left(\mathbb{N}^{2}\right)^{c}$ and $R\left(\mathbb{N}^{2}\right)=\ell_{2}\left(\mathbb{N}^{2}\right)^{r}$.

Lemma 2.4 There exists a subsequence $\left(f_{n_{k}}\right)$ of $\left(f_{k}\right)$ such that $\left(f_{k}\right)$ is completely equivalent to the basic sequence $\left(f_{n_{k}} \otimes e_{k}\right)$ in $\left(C\left(\mathbb{N}^{2}\right) \oplus R\left(\mathbb{N}^{2}\right)\right) / \ell_{2}(S)$.

Proof. Let $q: C \oplus R \rightarrow(C \oplus R) / S$ be the quotient map and $\left(\delta_{k}\right)$ a positive sequence. Since $\left(f_{k}\right)$ is a basic sequence in $(C \oplus R) / S$ and weakly converges to 0 , by a standard perturbation argument we find two increasing sequences $\left(n_{k}\right)$ and $\left(m_{k}\right)$ of positive integers such that

$$
\left\|f_{n_{k}}-\sum_{m_{k} \leq i<m_{k+1}}\left(\alpha_{i} q\left(e_{i 1}\right)+\beta_{i} q\left(e_{1 i}\right)\right)\right\|<\delta_{k}
$$

for some $a_{i}, \beta_{i} \in \mathbb{C}$. It follows that if the $\delta_{k}$ are sufficiently small, $\left(f_{n_{k}}\right)$ is 2 -completely equivalent to $\left(\tilde{f}_{n_{k}}\right)$, where

$$
\widetilde{f}_{n_{k}}=\sum_{m_{k} \leq i<m_{k+1}}\left(\alpha_{i} q\left(e_{i 1}\right)+\beta_{i} q\left(e_{1 i}\right)\right) .
$$

Replacing $\left(f_{n_{k}}\right)$ by $\left(\widetilde{f}_{n_{k}}\right)$ if necessary, we may assume $f_{n_{k}}=\widetilde{f}_{n_{k}}$ for all $k$. In this case we will say that the $f_{n_{k}}$ 's have disjoint supports. On the other hand, the complete symmetry of $\left(f_{k}\right)$ implies that $\left(f_{k}\right)$ is completely equivalent to $\left(f_{n_{k}}\right)$. Thus it remains to show that $\left(f_{n_{k}}\right)$ is completely equivalent to $\left(f_{n_{k}} \otimes e_{k}\right)$.

Given a finite sequence $\left(x_{k}\right) \subset S_{\infty}$ set

$$
x=\sum_{k} x_{k} \otimes f_{n_{k}} \quad \text { and } \quad \widetilde{x}=\sum_{k} x_{k} \otimes f_{n_{k}} \otimes e_{k} .
$$

We are going to show

$$
\|x\|_{S_{\infty}[(C \oplus R) / S]}=\|\widetilde{x}\|_{S_{\infty}\left[\left(C\left(\mathbb{N}^{2}\right) \oplus R\left(\mathbb{N}^{2}\right)\right) / \ell_{2}(S)\right]} .
$$

Let $a \in S_{\infty}[C]$ and $b \in S_{\infty}[R]$ such that $x=\mathrm{id} \otimes q(a, b)$. Now let $P_{k}$ be the projection onto the interval $\left[m_{k}, m_{k+1}\right)$. Namely, $P_{k}$ is the projection on $\ell_{2}$ such that $P_{k}\left(e_{i}\right)=e_{i}$ if $m_{k} \leq i<m_{k+1}$ and $P_{k}\left(e_{i}\right)=0$ otherwise. $P_{k}$ is viewed as projections on both $C$ and $R$ (recalling that we identify $\left(e_{i 1}\right)$ and $\left(e_{1 i}\right)$ with $\left.\left(e_{i}\right)\right)$. Since $P_{k}(S) \subset S$, thanks to the invariance of $S$ by diagonal operators, $P_{k}$ induces a projection $\widetilde{P}_{k}$ on $(C \oplus R) / S$. Then $q P_{k}=\widetilde{P}_{k} q$. Thus we find

$$
x_{k} \otimes f_{n_{k}}=\mathrm{id} \otimes \widetilde{P}_{k}(x)=\mathrm{id} \otimes q\left(\mathrm{id} \otimes P_{k}(a), \mathrm{id} \otimes P_{k}(b)\right) \stackrel{\text { def }}{=} \mathrm{id} \otimes q\left(\widetilde{a}_{k}, \widetilde{b}_{k}\right) .
$$

Therefore

$$
\widetilde{x}=\operatorname{id} \otimes q \otimes \operatorname{id}\left(\sum_{k} \widetilde{a}_{k} \otimes e_{k}, \sum_{k} \widetilde{b}_{k} \otimes e_{k}\right) \stackrel{\text { def }}{=} \operatorname{id} \otimes q \otimes \operatorname{id}(\widetilde{a}, \widetilde{b}) .
$$


It is clear that

$$
\|\widetilde{a}\|_{S_{\infty}\left[C\left(\mathbb{N}^{2}\right)\right]} \leq\|a\|_{S_{\infty}[C]} \quad \text { and } \quad\|\widetilde{b}\|_{S_{\infty}\left[R\left(\mathbb{N}^{2}\right)\right]} \leq\|b\|_{S_{\infty}[R]}
$$

whence

$$
\|\widetilde{x}\|_{S_{\infty}\left[\left(C\left(\mathbb{N}^{2}\right) \oplus R\left(\mathbb{N}^{2}\right)\right) / \ell_{2}(S)\right]} \leq\|x\|_{S_{\infty}[(C \oplus R) / S]} .
$$

Conversely, let $\widetilde{a} \in S_{\infty}\left[C\left(\mathbb{N}^{2}\right)\right], \widetilde{b} \in S_{\infty}\left[R\left(\mathbb{N}^{2}\right)\right]$ such that $\widetilde{x}=\operatorname{id} \otimes q \otimes \operatorname{id}(\widetilde{a}, \widetilde{b})$. As before, let $Q_{k}$ be the projection onto the subset $\left[m_{k}, m_{k+1}\right) \times\{k\}$ (considered as a projection on $\ell_{2}\left(\mathbb{N}^{2}\right)$ ). Put $a_{k}=Q_{k}(\widetilde{a})$ and $b_{k}=Q_{k}(\widetilde{b})$. We regard $a_{k}$ and $b_{k}$ as elements in $S_{\infty}[C]$ and $S_{\infty}[R]$, respectively. Then

$$
x_{k} \otimes f_{n_{k}}=\mathrm{id} \otimes q\left(a_{k}, b_{k}\right) .
$$

Thus

$$
x=\sum_{k} x_{k} \otimes f_{n_{k}}=\mathrm{id} \otimes q\left(\sum_{k} a_{k}, \sum_{k} b_{k}\right) \stackrel{\text { def }}{=} \mathrm{id} \otimes q(a, b) .
$$

It is easy to see

$$
\|a\|_{S_{\infty}[C]} \leq\|\widetilde{a}\|_{S_{\infty}\left[C\left(\mathbb{N}^{2}\right)\right]} \quad \text { and } \quad\|b\|_{S_{\infty}[R]} \leq\|\widetilde{b}\|_{S_{\infty}\left[R\left(\mathbb{N}^{2}\right)\right]}
$$

so

$$
\|x\|_{S_{\infty}[(C \oplus R) / S]} \leq\|\widetilde{x}\|_{S_{\infty}\left[\left(C\left(\mathbb{N}^{2}\right) \oplus R\left(\mathbb{N}^{2}\right)\right) / \ell_{2}(S)\right]} .
$$

Thus (2.4) is proved. Therefore, $\left(f_{n_{k}}\right)$ is 1-completely equivalent to $\left(f_{n_{k}} \otimes e_{k}\right)$.

Remark 2.5 The preceding proof shows that if the $f_{k}$ 's have disjoint supports, then $\left(f_{k}\right)$ is 1completely equivalent to $\left(f_{k} \otimes e_{k}\right)$.

We will use ultraproducts of operator spaces (see [P5]). If $\mathcal{U}$ is a free ultrafilter on $\mathbb{N}$ and $E$ an operator space, we denote by $E^{\mathcal{U}}$ the ultrapower $\prod_{\mathcal{U}} E$. Recall that $E$ is naturally identified as a subspace of $E^{\mathcal{U}}$. Let $H$ be the Hilbert space ultrapower of $\ell_{2}$ (in the category of Banach spaces). Then

$$
\left(\frac{C \oplus R}{S}\right)^{\mathcal{U}}=\frac{H^{c} \oplus H^{r}}{S^{\mathcal{U}}} .
$$

Consequently, $F^{\mathcal{U}}$ is a subspace of $\left(H^{c} \oplus H^{r}\right) / S^{\mathcal{U}}$. Let $\tilde{f}$ be the element of $F^{\mathcal{U}}$ represented by the basis $\left(f_{k}\right)$.

Lemma 2.6 For any finite sequence $\left(x_{k}\right) \subset S_{\infty}$ we have

$$
\left\|\sum_{k} x_{k} \otimes f_{k}\right\|_{S_{\infty}[F]} \sim_{c}\left\|\sum_{k} x_{k} \otimes \tilde{f} \otimes e_{k}\right\|_{S_{\infty}\left[\left(\left(\ell_{2}(H)\right)^{c} \oplus\left(\ell_{2}(H)\right)^{r}\right) / \ell_{2}\left(S^{\mathcal{U}}\right)\right]} .
$$

Proof. By Lemma 2.4, we can assume that the supports of the $f_{k}$ are disjoint. Then by the complete symmetry of $\left(f_{k}\right)$ and Lemma 2.4 (and also Remark 2.5), we find

$$
\left\|\sum_{k=1}^{m} x_{k} \otimes f_{k}\right\|_{S_{\infty}[F]}=\left\|\sum_{k=1}^{m} x_{k} \otimes f_{n_{k}} \otimes e_{k}\right\|_{S_{\infty}\left[\left(C\left(\mathbb{N}^{2}\right) \oplus R\left(\mathbb{N}^{2}\right)\right) / \ell_{2}(S)\right]}
$$

for any $x_{1}, \ldots, x_{m} \in S_{\infty}$ and any distinct positive integers $n_{1}, \ldots, n_{m}$. We can assume that the $x_{k}$ are finite matrices and that all direct sums are taken in the $\ell_{2}$-sense. Then we find

$$
\left\|\sum_{k=1}^{m} x_{k} \otimes f_{k}\right\|_{S_{\infty}[F]}=\lim _{n_{1}, \mathcal{U}} \cdots \lim _{n_{m}, \mathcal{U}}\left\|\sum_{k=1}^{m} x_{k} \otimes f_{n_{k}} \otimes e_{k}\right\|_{S_{\infty}\left[\left(C\left(\mathbb{N}^{2}\right) \oplus R\left(\mathbb{N}^{2}\right)\right) / \ell_{2}(S)\right]} .
$$

This implies the desired assertion. 
Proof of Theorem [2.3. Let $\left(S^{\mathcal{U}}\right)^{\perp}$ be the orthogonal complement of $S^{\mathcal{U}}$ in $\left(H^{c} \oplus H^{r}\right)^{*}=\bar{H}^{r} \oplus \bar{H}^{c}$. Then

$$
\left(\frac{H^{c} \oplus H^{r}}{S^{\mathcal{U}}}\right)^{*}=\left(S^{\mathcal{U}}\right)^{\perp} .
$$

On the other hand, by [X] we find four subspaces $\bar{K}_{j} \subset \bar{H}, 0 \leq j \leq 3$ and an injective closed densely defined operator $D: \bar{K}_{2} \rightarrow \bar{K}_{3}$ of dense range such that

$$
\left(S^{\mathcal{U}}\right)^{\perp}=\bar{K}_{0}^{r} \oplus \bar{K}_{1}^{c} \oplus \Gamma(D) .
$$

By the discussion following Theorem 2.3, we can assume that $K_{2}=K_{3}=K, D$ is a positive operator on $\bar{K}$ and $\bar{K}$ has an orthonormal basis $\left(\bar{g}_{i}\right)_{i \in I}$ of eigenvectors of $D$. The eigenvalue associated to $\bar{g}_{i}$ is denoted by $d_{i}$. We then deduce that

$$
\begin{aligned}
\frac{H^{c} \oplus H^{r}}{S^{\mathcal{U}}} & =\left(\left(S^{\mathcal{U}}\right)^{\perp}\right)^{*}=\left(\bar{K}_{0}^{r} \oplus \bar{K}_{1}^{c}\right)^{*} \oplus \Gamma(D)^{*} \\
& =K_{0}^{c} \oplus K_{1}^{r} \oplus \frac{K^{c} \oplus K^{r}}{\Gamma(D)^{\perp}}
\end{aligned}
$$

where $\Gamma(D)^{\perp}=\{(-D y, y): y \in \operatorname{Dom}(D)\}$. Note that $\Gamma(D)^{\perp}$ is the closed linear span of all $\left(-d_{i} g_{i}, g_{i}\right)$ in $K \oplus K$. Thus the operator space structure of $\left(K^{c} \oplus K^{r}\right) / \Gamma(D)^{\perp}$ is determined as follows. Let $q: K^{c} \oplus K^{r} \rightarrow\left(K^{c} \oplus K^{r}\right) / \Gamma(D)^{\perp}$ be the quotient map. Then $\left(q\left(g_{i}, 0\right)\right)_{i \in I}$ is a basis of $\left(K^{c} \oplus K^{r}\right) / \Gamma(D)^{\perp}$. For any finite family $\left(x_{i}\right) \subset S_{\infty}$

$$
\left\|\sum_{i} x_{i} \otimes q\left(g_{i}, 0\right)\right\|_{S_{\infty}\left[\left(K^{c} \oplus K^{r}\right) / \Gamma(D)^{\perp}\right]}=\inf _{x_{i}=a_{i}+b_{i}} \max \left(\left\|\sum_{i} a_{i}^{*} a_{i}\right\|^{1 / 2},\left\|\sum_{i} d_{i}^{-2} b_{i} b_{i}^{*}\right\|^{1 / 2}\right) .
$$

Since $\tilde{f} \in F^{\mathcal{U}} \subset\left(H^{c} \oplus H^{r}\right) / S^{\mathcal{U}}$ and $\|\widetilde{f}\|=1$, there exist $\widetilde{f}_{0} \in K_{0}^{c}, \widetilde{f}_{1} \in K_{1}^{r}$ and $\widetilde{f}_{2} \in\left(K^{c} \oplus\right.$ $\left.K^{r}\right) / \Gamma(D)^{\perp}$ such that

$$
\widetilde{f}=\widetilde{f}_{0}+\widetilde{f}_{1}+\widetilde{f}_{2} \quad \text { and } \quad\left\|\widetilde{f}_{j}\right\| \leq 1, \quad j=0,1,2 .
$$

On the other hand, by the previous decomposition of $\left(H^{c} \oplus H^{r}\right) / S^{\mathcal{U}}$ we find

$$
\frac{\left(\ell_{2}(H)\right)^{c} \oplus\left(\ell_{2}(H)\right)^{r}}{\ell_{2}\left(S^{\mathcal{U}}\right)}=\left(\ell_{2}\left(K_{0}\right)\right)^{c} \oplus\left(\ell_{2}\left(K_{1}\right)\right)^{r} \oplus \frac{\left(\ell_{2}(K)\right)^{c} \oplus\left(\ell_{2}(K)\right)^{r}}{\ell_{2}\left(\Gamma(D)^{\perp}\right)} .
$$

Then for any finite sequence $\left(x_{k}\right) \subset S_{\infty}$, by Lemma 2.6 we deduce

$$
\begin{aligned}
\left\|\sum_{k} x_{k} \otimes f_{k}\right\|_{S_{\infty}[F]} \sim_{c} \max & \left(\left\|\widetilde{f}_{0}\right\|\left\|\sum_{k} x_{k}^{*} x_{k}\right\|^{1 / 2},\left\|\widetilde{f}_{1}\right\|\left\|\sum_{k} x_{k} x_{k}^{*}\right\|^{1 / 2},\right. \\
& \left.\left\|\sum_{k} x_{k} \otimes \widetilde{f}_{2} \otimes e_{k}\right\|_{S_{\infty}\left[\left(\left(\ell_{2}(K)\right)^{c} \oplus\left(\ell_{2}(K)\right)^{r}\right) / \ell_{2}\left(\Gamma(D)^{\perp}\right)\right]}\right) .
\end{aligned}
$$

Now write $\widetilde{f}_{2}=\left(\alpha_{i}, \beta_{i}\right)_{i \in I}+\Gamma(D)^{\perp}$ with

$$
\sum_{i}\left(\left|\alpha_{i}\right|^{2}+\left|\beta_{i}\right|^{2}\right) \leq 2\left\|\widetilde{f}_{2}\right\|^{2} \leq 2
$$

Thus at most countably many $\left(\alpha_{i}, \beta_{i}\right)$ 's are nonzero. On the other hand, if $\alpha_{i}=-d_{i} \beta_{i}$ for some $i$, then $\left(\alpha_{i} g_{i}, \beta_{i} g_{i}\right) \in \Gamma(D)^{\perp}$; so this term does not contribute to $\widetilde{f}_{2}$. Hence, without loss of generality we can assume that the index set $I$ is equal to $\mathbb{N}$ and $\alpha_{i} \neq-d_{i} \beta_{i}$ for every $i$. Let $\widehat{a}=\left(\widehat{a}_{i k}\right) \in S_{\infty}\left[\left(\ell_{2}(K)\right)^{c}\right]$ and $\widehat{b}=\left(\widehat{b}_{i k}\right) \in S_{\infty}\left[\left(\ell_{2}(K)\right)^{r}\right]$ such that

$$
\sum_{k} x_{k} \otimes \widetilde{f}_{2} \otimes e_{k}=\mathrm{id} \otimes q \otimes \operatorname{id}(\widehat{a}, \widehat{b}) .
$$


Note that $\widehat{a}_{i k}, \widehat{b}_{i k} \in S_{\infty}$, the indices $i$ and $k$ correspond to the bases $\left(g_{i}\right)$ of $K$ and $\left(e_{k}\right)$ of $\ell_{2}$, respectively. Then

$$
\alpha_{i} x_{k}-\widehat{a}_{i k}=-d_{i}\left(\beta_{i} x_{k}-\widehat{b}_{i k}\right), \quad \forall i, k ;
$$

whence

$$
x_{k}=\frac{1}{\alpha_{i}+d_{i} \beta_{i}} \widehat{a}_{i k}+\frac{d_{i}}{\alpha_{i}+d_{i} \beta_{i}} \widehat{b}_{i k} \stackrel{\text { def }}{=} a_{i k}+b_{i k} .
$$

Let

$$
u_{c}(i)=\left|\alpha_{i}+d_{i} \beta_{i}\right|^{2} \quad \text { and } \quad u_{r}(i)=d_{i}^{-2}\left|\alpha_{i}+d_{i} \beta_{i}\right|^{2} .
$$

Then

$$
\max \left(\left\|\sum_{i, k} u_{c}(i) a_{i k}^{*} a_{i k}\right\|^{1 / 2},\left\|\sum_{i, k} u_{r}(i) b_{i k} b_{i k}^{*}\right\|^{1 / 2}\right)=\max \left(\|\widehat{a}\|_{S_{\infty}\left[\left(\ell_{2}(K)\right)^{c}\right]},\|\widehat{b}\|_{S_{\infty}\left[\left(\ell_{2}(K)\right)^{r}\right]}\right) .
$$

Therefore, we deduce

$$
\begin{aligned}
& \left\|\sum_{k} x_{k} \otimes \tilde{f}_{2} \otimes e_{k}\right\|_{S_{\infty}\left[\left(\left(\ell_{2}(K)\right)^{c} \oplus\left(\ell_{2}(K)\right)^{r}\right) / \ell_{2}(\Gamma(D) \perp)\right]} \\
& \quad=\inf _{x_{k}=a_{i k}+b_{i k}} \max \left(\left\|\left(\sum_{i, k} u_{c}(i) a_{i k}^{*} a_{i k}\right)^{1 / 2}\right\|,\left\|\left(\sum_{i, k} u_{r}(i) b_{i k} b_{i k}^{*}\right)^{1 / 2}\right\|\right) .
\end{aligned}
$$

It remains to check the weight condition (2.3). This is easy. Indeed,

$$
\min \left(u_{c}(i), u_{r}(i)\right) \leq 2 \min \left(\alpha_{i}^{2}+d_{i}^{2} \beta_{i}^{2}, d_{i}^{-2} \alpha_{i}^{2}+\beta_{i}^{2}\right) \leq 2\left(\alpha_{i}^{2}+\beta_{i}^{2}\right) .
$$

It follows that

$$
\sum_{i} \min \left(u_{c}(i), u_{r}(i)\right) \leq 2 \sum_{i}\left(\alpha_{i}^{2}+\beta_{i}^{2}\right) \leq 4
$$

Therefore, the theorem is proved.

Corollary 2.7 Every infinite dimensional space $F \in H Q S(C \oplus R)$ is completely isomorphic to $C, R, C \cap R$ or $K_{u_{c}, u_{r}}$ for some positive sequences $u_{c}$ and $u_{r}$. Moreover, in each case we have $C \cap R \subset F \subset C+R$, up to complete isomorphism.

Proof. Let $F$ be represented as in Theorem 2.3. Then

$$
\lambda C \cap \gamma R \cap K_{u_{c}, u_{r}}= \begin{cases}C \cap R & \text { if } \lambda>0, \gamma>0 \\ C & \text { if } \lambda>0, \gamma=0 \\ R & \text { if } \lambda=0, \gamma>0 \\ K_{u_{c}, u_{r}} & \text { if } \lambda=0, \gamma=0\end{cases}
$$

To show the second part it suffices to prove $C \cap R \subset K_{u_{c}, u_{r}} \subset C+R$. Let $\left(x_{k}\right) \subset S_{\infty}$ be a finite sequence. Consider the decomposition $x_{k}=a_{i k}+b_{i k}$ given by

$$
a_{i k}=\frac{u_{r}(i)}{u_{c}(i)+u_{r}(i)} x_{k} \quad \text { and } \quad b_{i k}=\frac{u_{c}(i)}{u_{c}(i)+u_{r}(i)} x_{k} .
$$

Then

$$
\sum_{i, k} u_{c}(i) a_{i k}^{*} a_{i k}=\sum_{i} \frac{u_{c}(i) u_{r}(i)}{u_{c}(i)+u_{r}(i)} \sum_{k} x_{k}^{*} x_{k} .
$$

The first sum on the right hand side is finite by virtue of (2.3). We have a similar formula for the row case. It thus follows that $C \cap R \subset K_{u_{c}}, u_{r}$. To show the second inclusion take a decomposition $x_{k}=a_{i k}+b_{i k}$. Let

$$
\widehat{a}_{k}=\frac{1}{\mu} \sum_{i} \frac{u_{c}(i) u_{r}(i)}{u_{c}(i)+u_{r}(i)} a_{i k} \quad \text { and } \quad \widehat{b}_{k}=\frac{1}{\mu} \sum_{i} \frac{u_{c}(i) u_{r}(i)}{u_{c}(i)+u_{r}(i)} b_{i k}
$$


where

$$
\mu=\sum_{i} \frac{u_{c}(i) u_{r}(i)}{u_{c}(i)+u_{r}(i)}
$$

Then $x_{k}=\widehat{a}_{k}+\widehat{b}_{k}$. On the other hand, by the convexity of the operator square function we find

$$
\widehat{a}_{k}^{*} \widehat{a}_{k} \leq \frac{1}{\mu^{2}} \sum_{i} \frac{u_{c}(i) u_{r}(i)^{2}}{\left(u_{c}(i)+u_{r}(i)\right)^{2}} \sum_{i} u_{c}(i) a_{i k}^{*} a_{i k} \leq \frac{1}{\mu} \sum_{i} u_{c}(i) a_{i k}^{*} a_{i k} .
$$

Using similar inequalities for the row terms, we deduce the inclusion $K_{u_{c}, u_{r}} \subset C+R$.

Remark 2.8 Let $u_{c}$ and $u_{r}$ be two weights on $(\Omega, \nu)$ verifying (2.1). Using the arguments in the proof of Corollary 2.7, one easily checks the following properties:

(i) If

$$
\int_{\Omega} u_{c} d \nu<\infty \text { and } \int_{\Omega} u_{r} d \nu<\infty
$$

then $K_{u_{c}, u_{r}}=C+R$.

(ii) If $A \subset \Omega$ is such that

$$
\int_{A} u_{c} d \nu<\infty \text { and } \int_{A} u_{r} d \nu<\infty
$$

then the parts of $u_{c}$ and $u_{r}$ on $A$ do not contribute to $K_{u_{c}, u_{r}}$. More precisely, $K_{u_{c}, u_{r}}$ is completely isomorphic to $K_{\widetilde{u}_{c}, \widetilde{u}_{r}}$, constructed on $A^{c}$ relative to the weights $\widetilde{u}_{c}=\left.u_{c}\right|_{A^{c}}$ and $\widetilde{u}_{r}=\left.u_{r}\right|_{A^{c}}$.

Theorem 2.3 can be extended to a more general setting. Let $X$ and $Y$ be two homogeneous Hilbertian operator spaces. Let $\left(u_{c}, u_{r}\right)$ be a pair of weights on $(\Omega, \nu)$ satisfying (2.1). We consider the space

$$
G_{u_{c}, u_{r}}(X, Y)=L_{2}\left(u_{c} ; X\right)^{X}+L_{2}\left(u_{r} ; Y\right)^{Y},
$$

where $L_{2}\left(u_{c} ; X\right)^{X}$ denotes $L_{2}\left(u_{c} ; X\right)$ equipped with the operator space structure of $X$ and similarly for $L_{2}\left(u_{r} ; Y\right)^{Y}$. Let $K_{u_{c}, u_{r}}(X, Y)$ be the constant function subspace of $G_{u_{c}, u_{r}}(X, Y)$. Reexamining its proof, we find that Theorem 2.3 is still valid with $(C, R)$ replaced by $(X, Y)$. Let us record this in the following statement, which is particularly interesting when $X=C_{p}$ and $Y=R_{p}$.

Theorem 2.9 Let $X$ and $Y$ be two separable infinite dimensional homogeneous Hilbertian operator spaces. Let $F$ be an infinite dimensional homogeneous space in $Q S(X \oplus Y)$. Then there exist two constants $\lambda, \gamma \in[0,1]$ and two positive sequences $u_{c}=\left(u_{c}(j)\right)_{j \geq 1}, u_{r}=\left(u_{r}(j)\right)_{j \geq 1}$ such that $u_{c}$ and $u_{r}$ satisfy (2.3) and such that $F$ is completely isomorphic to $\lambda X \cap \gamma Y \cap K_{u_{c}, u_{r}}(X, Y)$. Moreover, the equivalence constants depend only on the homogeneity and Hilbertian constants of $X, Y$ and $F$.

\section{Homogeneous spaces satisfying a regularity condition}

The representation of a space $F \in H Q S(C \oplus R)$ given by Theorem 2.3 is far from being unique. The objective of this section is to give a certain regularity condition on $\left(u_{c}, u_{r}\right)$ which ensures that if $F=K_{u_{c}}, u_{r}$, then up to equivalence $u_{c}$ and $u_{r}$ are uniquely determined by $F$. The first step towards this is the following simple result. In the sequel $\mathbb{R}$ will be always equipped with Lebesgue measure. 
Proposition 3.1 Let $\left(u_{c}, u_{r}\right)$ be a pair of weights on $(\Omega, \nu)$ satisfying (2.1). Then $K_{u_{c}, u_{r}}$ is completely isomorphic to $K_{w_{c}, w_{r}}$, where $w_{c}$ and $w_{r}$ are two weights on $\mathbb{R}$ satisfying the following conditions

$$
\left\{\begin{array}{l}
w_{c}=1 \text { on } \mathbb{R}_{+}, w_{c} \text { is nondecreasing and left continuous; } \\
w_{r}=1 \text { on } \mathbb{R}_{-}, w_{c} \text { is nonincreasing and right continuous; } \\
\int_{-\infty}^{0} w_{c}(s) d s=1=\int_{0}^{\infty} w_{r}(s) d s .
\end{array}\right.
$$

Proof. Consider the following partition of $\Omega$ :

$$
A_{k}=\left\{\omega \in \Omega: 2^{-k-1} \leq \frac{u_{r}(\omega)}{u_{c}(\omega)}<2^{-k}\right\}, \quad k \in \mathbb{Z}
$$

Let

$$
\widetilde{u}_{c}(k)=u_{c}\left(A_{k}\right) \quad \text { and } \quad \widetilde{u}_{r}(k)=2^{-k} u_{c}\left(A_{k}\right) \quad k \in \mathbb{Z},
$$

where $u_{c}(A)=\int_{A} u_{c} d \nu$ for $A \subset \Omega$. By Proposition 2.2, $K_{u_{c}, u_{r}}$ is completely isomorphic to $K_{\widetilde{u}_{c}, \widetilde{u}_{r}}$. Now put

$$
s_{0}=0, \quad s_{-1}=-1 \quad \text { and } \quad s_{k}=-u_{r}\left(A_{k+1}\right)-u_{r}\left(A_{k+2}\right)-\cdots-u_{r}\left(A_{-1}\right)-1 \text { for } k \leq-2 .
$$

Define

$$
w_{c}(s)=\frac{1}{2 \lambda} \sum_{k \leq-2} 2^{k} \mathbb{1}_{\left[s_{k}, s_{k+1}\right]}(s) \text { for } s \in(-\infty,-1]
$$

where

$$
\lambda=\max \left(1, \int_{\Omega} \min \left(u_{c}, u_{r}\right) d \nu\right) .
$$

Then

$$
\begin{aligned}
\int_{-\infty}^{-1} w_{c}(s) d s & =\frac{1}{2 \lambda} \sum_{k \leq-2} 2^{k} u_{r}\left(A_{k+1}\right) \\
& \leq \frac{1}{2 \lambda} \sum_{k \leq-2} \int_{A_{k+1}} u_{c} d \nu \leq \frac{1}{2 \lambda} \int_{\Omega} \min \left(u_{c}, u_{r}\right) d \nu \leq \frac{1}{2}
\end{aligned}
$$

Completing $w_{c}$ by

$$
w_{c}(s)=1-\int_{-\infty}^{-1} w_{c}(s) d s \text { for } s \in(-1,0] \text { and } w_{c}(s)=1 \text { for } s>0,
$$

we see that $w_{c}$ is nondecreasing and

$$
\int_{-\infty}^{0} w_{c}(s) d s=1
$$

The second function $w_{r}$ is defined similarly by using $u_{r}$ instead of $u_{c}$. Indeed, letting

$$
s_{1}=1 \quad \text { and } \quad s_{k}=1+u_{c}\left(A_{1}\right)+\cdots+u_{c}\left(A_{k-1}\right) \text { for } k \geq 2,
$$

we define $w_{r}$ by

$$
w_{r}=\mathbb{1}_{(-\infty, 0)}+\left(1-\int_{1}^{\infty} w_{r}(s) d s\right) \mathbb{1}_{[0,1)}+\frac{1}{2 \lambda} \sum_{k \geq 1} 2^{-k-1} \mathbb{1}_{\left[s_{k}, s_{k+1}\right)} .
$$

Then by Proposition 2.2 (and Remark 2.8), $K_{w_{c}, w_{r}}$ is completely isomorphic to $K_{\widetilde{u}_{c}, \widetilde{u}_{c}}$, so to $K_{u_{c}, u_{r}}$ too. 
Remark 3.2 The last condition in (3.1) is not essential for $K_{w_{c}, w_{r}}$. What really matters is the behavior of the weights at infinity. More precisely, if $v_{c}$ and $v_{r}$ are two other locally integrable weights on $\mathbb{R}$ such that

$$
c_{1} v_{c}(s) \leq w_{c}(s) \leq c_{2} v_{c}(s) \quad \text { and } \quad c_{1} v_{r}(s) \leq w_{r}(s) \leq c_{2} v_{r}(s)
$$

for two positive constants $c_{1}, c_{2}$ and for all $s \in \mathbb{R}$ with $|s|$ sufficiently big, then $K_{w_{c}, w_{r}}$ and $K_{v_{c}, v_{r}}$ are completely isomorphic. In particular, if $w_{c}$ and $w_{r}$ satisfy the two first conditions in (3.1) and are integrable on $\mathbb{R}_{-}$and $\mathbb{R}_{+}$, respectively, then we can adjust them, up to equivalence, so that they satisfy the last condition too. This is achieved by the arguments in the proof of Proposition 3.1 and Remark 2.8,

In the following we will often assume that $w_{c}$ and $w_{r}$ satisfy (3.1). Note that the left (right) continuity is assumed only for convenience. In fact, by perturbation and Remark 3.2 we can even assume that $w_{c}$ and $w_{r}$ are continuous. For the same reason, we can also assume that $w_{c}$ (resp. $\left.w_{r}\right)$ is increasing on $\mathbb{R}_{-}$(resp. decreasing on $\mathbb{R}_{+}$). On the other hand, if $w_{r}=0$ on $\left[s_{0}, \infty\right)$ for some $s_{0} \geq 0$, then by Remarks 2.1 and 2.8 we need only to consider the part on $\mathbb{R}_{-}$. Thus for presentation simplicity we will assume that both $w_{c}$ and $w_{r}$ are strictly positive on $\mathbb{R}$ in the sequel.

We will show that under a certain regularity condition $w_{c}$ and $w_{r}$ are uniquely determined, up to equivalence at infinity, by the operator space structure of $K_{w_{c}}, w_{r}$, i.e., by the fundamental functions of $K_{w_{c}, w_{r}}$. To this end we require some preparations and two auxiliary pairs of functions associated with $\left(w_{c}, w_{r}\right)$. We will concentrate only on $w_{r}$ since $w_{c}$ can be dealt with by using the symmetry $t \mapsto-t$.

Let $w$ be an integrable nonincreasing right continuous positive function on $\mathbb{R}_{+}$. Consider the functions $h$ and $g$ on $\mathbb{R}_{+}$defined by

$$
h(t)=\int_{t}^{\infty} w(s) d s \quad \text { and } \quad g(s)=\inf _{t>0} \max (t, s h(t)) .
$$

The following is entirely elementary.

Lemma 3.3 Let $w, h, g$ be as above.

(i) $h$ is decreasing, continuous and derivable from right with $-w$ as right derivative.

(ii) $g$ is increasing, continuous and $g(s) \rightarrow \infty$ as $s \rightarrow \infty$.

(iii) For any $s \in \mathbb{R}_{+}$the infimum defining $g(s)$ is attained at a unique $t$ such that $t=\operatorname{sh}(t)$. Moreover (with $g^{-1}$ denoting the inverse of $g$ ),

$$
\frac{h(t)}{t}=\frac{1}{g^{-1}(t)}, \quad \forall t>0
$$

Proof. (i) and (ii) are obvious except the injectivity of $g$ which will follow from (iii). To prove (iii) we observe that by the continuity of $h$ and the fact that $h(t) \rightarrow 0$ as $t \rightarrow \infty$, for any $s \in \mathbb{R}_{+}$there exists $t$ such that $g(s)=\max (t, \operatorname{sh}(t))$. If $t<s h(t)$, then by the continuity of $h$ once more there exists $t_{1}$ such that $t_{1}>t$ and $t_{1}<s h\left(t_{1}\right)$; whence

$$
g(s) \leq \max \left(t_{1}, \operatorname{sh}\left(t_{1}\right)\right)=\operatorname{sh}\left(t_{1}\right)<\operatorname{sh}(t)=\max (t, \operatorname{sh}(t))=g(s),
$$

which is a contradiction. Similarly, $t>\operatorname{sh}(t)$ cannot happen. Therefore, $t=s h(t)$; so $g(s)=t$ and $g(s)=s h(t)$. This implies in particular that $g$ is injective, so increasing. Consequently, its inverse $g^{-1}$ exists and $s=g^{-1}(t)$. Together with $t=s h(t)$, this yields (3.3). 
We will employ Orlicz space techniques, one of the main novelties of the paper. Recall that an Orlicz function is a convex function $\varphi$ on $[0, \infty)$ with $\varphi(0)=0$. The Orlicz sequence space $\ell_{\varphi}$ consists of all complex sequences $x=\left(x_{n}\right)$ such that

$$
\sum_{n} \varphi\left(\frac{\left|x_{n}\right|}{\lambda}\right)<\infty
$$

for some $\lambda>0$ and is equipped with the norm

$$
\|x\|_{\varphi}=\inf \left\{\lambda>0: \sum_{n} \varphi\left(\frac{\left|x_{n}\right|}{\lambda}\right) \leq 1\right\} .
$$

Note that $\ell_{\varphi}$ depends, up to an equivalent norm, only on the values of $\varphi$ in a neighborhood of 0 . It is well-known that an Orlicz function $\varphi$ is uniquely determined, up to equivalence at 0 , by its fundamental sequence $\left(\varphi_{n}\right)_{n \geq 1}$, where

$$
\varphi_{n}=\left\|\sum_{k \leq n} e_{k}\right\|_{\varphi}=\frac{1}{\varphi^{-1}\left(\frac{1}{n}\right)}
$$

This is equivalent to saying that up to an equivalent norm, $\ell_{\varphi}$ is uniquely determined by $\left(\varphi_{n}\right)$ (see [LT, Proposition 4.a.5]). Here $\varphi^{-1}$ denotes the generalized inverse of $\varphi$, i.e., $\varphi^{-1}(t)=\sup \{s$ : $\varphi(s) \leq t\}$. Note that $\varphi$ is invertible except in the trivial case where $\varphi$ vanishes in a neighborhood of 0 .

Now let $\left(w_{c}, w_{r}\right)$ be a pair of positive weights on $\mathbb{R}$ satisfying (3.1). The functions $h$ and $g$ associated to $w_{r}$ as in (3.2) will be denoted by $h_{r}$ and $g_{r}$, respectively. Accordingly, the two functions on $\mathbb{R}_{+}$associated to the function $t \mapsto w_{c}(-t)$ will be denoted by $h_{c}$ and $g_{c}$. Define $\varphi_{c}, \varphi_{r}: \mathbb{R}_{+} \rightarrow \mathbb{R}_{+}$by

$$
\varphi_{c}(t)=t^{2} h_{c}\left(\frac{1}{t^{2}}\right) \quad \text { and } \quad \varphi_{r}(t)=t^{2} h_{r}\left(\frac{1}{t^{2}}\right)
$$

(with $\varphi_{c}(0)=\varphi_{r}(0)=0$ ). It is easy to check that $\varphi_{c}$ and $\varphi_{r}$ are convex functions. Indeed, using the equality

$$
\varphi_{r}(t)=t^{2} \int_{t^{-2}}^{\infty} w_{r}(s) d s
$$

we find

$$
\varphi_{r}^{\prime \prime}(t)=2 \int_{t^{-2}}^{\infty} w_{r}(s) d s+\frac{2}{t^{2}} w_{r}\left(\frac{1}{t^{2}}\right)-\frac{4}{t^{4}} w_{r}^{\prime}\left(\frac{1}{t^{2}}\right),
$$

where the derivatives are right derivatives. Since $w_{r}$ is nonincreasing, $\varphi_{r}^{\prime \prime}(t) \geq 0$, so $\varphi_{r}$ is convex.

The next lemma contains the main idea of our uniqueness theorem on $K_{w_{c}, w_{r}}$ and also explains the reason for the introduction of $h_{r}$ and $g_{r}$. Its proof uses an Orlicz space argument which will also play a key role in the proof of Theorem 4.2 below. We will consider only the diagonal elements of $R\left[K_{w_{c}}, w_{r}\right]$ although we can determine the norm of any element (see the proof of Theorem 4.2 for more details). Recall that $\left(e_{k}\right)$ denotes the canonical basis of $K_{w_{c}}, w_{r}$ and $\left(e_{k 1}\right)$ (resp. $\left.\left(e_{1 k}\right)\right)$ that of $C$ (resp. $R$ ).

Lemma 3.4 For any finite sequence $\left(x_{k}\right) \subset \mathbb{C}$ we have

$$
\left\|\sum_{k} x_{k} e_{1 k} \otimes e_{k}\right\|_{R\left[K_{\left.w_{c}, w_{r}\right]}\right.} \sim_{c}\left\|\left(x_{k}\right)\right\|_{\varphi_{r}} .
$$

Moreover, for any $n \in \mathbb{N}$

$$
\left\|\sum_{k=1}^{n} e_{1 k} \otimes e_{k}\right\|_{R\left[K_{\left.w_{c}, w_{r}\right]}\right]}^{2} \sim_{c} g_{r}(n) .
$$

A similar statement holds for the column case. 
Proof. We consider only the row case, the column part being treated similarly. We will use the following auxiliary function

$$
\mathrm{h}_{r}(t)=\inf \left\{w_{r}\left(A^{c}\right): A \subset \mathbb{R}, w_{c}(A) \leq t\right\}, \quad t>0 .
$$

We claim that

$$
h_{r}(t) \leq \mathrm{h}_{r}(t) \quad \text { and } \quad \mathrm{h}_{r}(1+t) \leq h_{r}(t)
$$

Indeed, first observe that

$$
\sup \left\{w_{r}(A): A \subset \mathbb{R}_{+},|A| \leq t\right\}=\int_{0}^{t} w_{r}(s) d s
$$

for $w_{r}$ is nonincreasing, where $|A|$ denotes the Lebesgue measure of $A$. Then we find

$$
\begin{aligned}
h_{r}(t) & =\int_{t}^{\infty} w_{r}(s) d s=1-\int_{0}^{t} w_{r}(s) d s \\
& =\inf \left\{w_{r}\left(A^{c} \cap \mathbb{R}_{+}\right): A \subset \mathbb{R},\left|A \cap \mathbb{R}_{+}\right| \leq t\right\} \\
& =\inf \left\{w_{r}\left(A^{c} \cap \mathbb{R}_{+}\right): A \subset \mathbb{R}, w_{c}\left(A \cap \mathbb{R}_{+}\right) \leq t\right\} .
\end{aligned}
$$

Therefore, $h_{r} \leq \mathrm{h}_{r}$. Since $w_{c}\left(\mathbb{R}_{-}\right)=1$, we also have $\mathrm{h}_{r}(1+t) \leq h_{r}(t)$.

Let $\phi_{r}(t)=t^{2} \mathrm{~h}_{r}\left(t^{-2}\right)$. Then the preceding claim shows that $\varphi_{r}$ and $\phi_{r}$ are equivalent at 0 with universal equivalence constants. Thus we need only to prove (3.4) with $\phi_{r}$ in place of $\varphi_{r}$. To this end first recall the following elementary identifications

$$
R\left[\ell_{2}^{c}\right]=R[C]=\mathbb{K}\left(\ell_{2}\right) \quad \text { and } \quad R\left[\ell_{2}^{r}\right]=R[R]=\left(S_{2}\right)^{r},
$$

where $\mathbb{K}\left(\ell_{2}\right)$ denotes the space of compact operators on $\ell_{2}$. Then we find

$$
R\left[G_{w_{c}, w_{r}}\right]=R\left[L_{2}^{c}\left(w_{c} ; \ell_{2}\right)\right]+R\left[L_{2}^{r}\left(w_{r} ; \ell_{2}\right)\right]=L_{2}^{c}\left(w_{c}\right) \otimes_{\min } \mathbb{K}\left(\ell_{2}\right)+L_{2}^{r}\left(w_{r} ; S_{2}\right) .
$$

Since we are dealing only with diagonal elements of $R\left[K_{w_{c}, w_{r}}\right]$, we use the diagonal projection $D$ on $\mathbb{K}\left(\ell_{2}\right)$ and $S_{2}$. The complete contractivity of $D$ on $\mathbb{K}\left(\ell_{2}\right)$ implies that id $\otimes D$ is a contraction on $L_{2}^{c}\left(w_{c}\right) \otimes_{\min } \mathbb{K}\left(\ell_{2}\right)$. On the other hand, it is trivial that id $\otimes D$ is also a contraction on $L_{2}^{r}\left(w_{r} ; S_{2}\right)$. Therefore, id $\otimes D$ is a contractive projection from $R\left[G_{w_{c}, w_{r}}\right]$ onto its diagonal part, which is $L_{2}^{c}\left(w_{c}\right) \otimes_{\min } c_{0}+\left(\ell_{2}\left(L_{2}\left(w_{r}\right)\right)^{r}\right.$. Since $R\left[K_{w_{c}, w_{r}}\right]$ is the constant function subspace of $R\left[G_{w_{c}, w_{r}}\right]$, for any finite sequence $x=\left(x_{k}\right) \subset \mathbb{C}$ we then deduce

$$
\left\|\sum_{k} x_{k} e_{1 k} \otimes e_{k}\right\|_{R\left[K_{w_{c}, w_{r}}\right]}=\inf \left\{\sup _{k}\left\|a_{k}\right\|_{L_{2}\left(w_{c}\right)}+\left(\sum_{k}\left\|b_{k}\right\|_{L_{2}\left(w_{r}\right)}^{2}\right)^{1 / 2}\right\}
$$

where the infimum runs over all decompositions $x_{k}=a_{k}+b_{k}$ a.e. on $\mathbb{R}$ with $a_{k} \in L_{2}\left(w_{c}\right)$ and $b_{k} \in L_{2}\left(w_{r}\right)$. Put

$$
\||x|\|=\inf \left\{\sup _{k}\left|x_{k}\right| w_{c}\left(A_{k}\right)^{1 / 2}+\left(\sum_{k}\left|x_{k}\right|^{2} w_{r}\left(A_{k}^{c}\right)\right)^{1 / 2}: A_{k} \subset \mathbb{R}\right\} .
$$

It is then easy to check that

$$
\frac{1}{2}\|x\| \leq\left\|\sum_{k} x_{k} e_{1 k} \otimes e_{k}\right\|_{R\left[K_{w_{c}, w_{r}}\right]} \leq\|\| x \| .
$$

Indeed, given a decomposition $x_{k}=a_{k}+b_{k}$ as above, define

$$
A_{k}=\left\{s \in \mathbb{R}:\left|a_{k}(s)\right| \geq \frac{\left|x_{k}\right|}{2}\right\} .
$$


Then $\left|b_{k}\right|>\left|x_{k}\right| / 2$ on $A_{k}^{c}$. Thus

$$
\frac{1}{2}\|x\| \leq\left\|\sum_{k} x_{k} e_{1 k} \otimes e_{k}\right\|_{R\left[K_{w_{c}, w_{r}}\right]} .
$$

The inverse inequality follows by taking the special decomposition $x_{k}=x_{k} \mathbb{1}_{A_{k}}+x_{k} \mathbb{1}_{A_{k}^{c}}$.

Now assume $\left\|\sum_{k=1}^{n} x_{k} e_{1 k} \otimes e_{k}\right\|_{R\left[K_{w_{c}, w_{r}}\right]}<1 / 2$. Without loss of generality, assume that $x_{k} \neq 0$ for every $1 \leq k \leq n$. Then we find $A_{k} \subset \mathbb{R}$ such that

$$
\sup _{k}\left|x_{k}\right| w_{c}\left(A_{k}\right)^{1 / 2}+\left(\sum_{k}\left|x_{k}\right|^{2} w_{r}\left(A_{k}^{c}\right)\right)^{1 / 2}<1 .
$$

Therefore,

$$
w_{c}\left(A_{k}\right) \leq \frac{1}{\left|x_{k}\right|^{-2}}, \quad \forall 1 \leq k \leq n
$$

and

$$
\sum_{k=1}^{n} \phi_{r}\left(\left|x_{k}\right|\right)=\sum_{k=1}^{n}\left|x_{k}\right|^{2} \mathrm{~h}_{r}\left(\frac{1}{\left|x_{k}\right|^{-2}}\right) \leq \sum_{k}\left|x_{k}\right|^{2} w_{r}\left(A_{k}^{c}\right) \leq 1 .
$$

Thus $\left\|\left(x_{k}\right)\right\|_{\phi_{r}} \leq 1$. Conversely, assume that $\left\|\left(x_{k}\right)\right\|_{\phi_{r}}<1$. Then there exist $A_{k} \subset \mathbb{R}$ such that

$$
w_{c}\left(A_{k}\right) \leq \frac{1}{\left|x_{k}\right|^{-2}} \quad \text { and } \quad \sum_{k}\left|x_{k}\right|^{2} w_{r}\left(A_{k}^{c}\right) \leq 1
$$

It follows that $\||x \|| \leq 2$; so $\| \sum_{k=1}^{n} x_{k} e_{1 k} \otimes e_{k} \|_{R\left[K_{w_{c}, w_{r}}\right]} \leq 2$. Therefore, (3.4) is proved. On the other hand, (3.3) implies

$$
\frac{1}{\sqrt{g_{r}(s)}}=\varphi^{-1}\left(\frac{1}{s}\right), \quad \forall s>0 .
$$

(3.5) is then a particular case of (3.4) since

$$
\left\|\sum_{k=1}^{n} e_{k}\right\|_{\varphi_{r}}=\frac{1}{\varphi_{r}^{-1}\left(\frac{1}{n}\right)}=\sqrt{g_{r}(n)}
$$

Let $F=K_{w_{c}, w_{r}}$. Then (3.5) shows that $R[F]$ is determined (up to equivalence) by $g_{r}$, so by $w_{r}$ too. In order to reverse this procedure, i.e., to determine $w_{r}$ by $R[F]$, we must know how to recover $w_{r}$ from $g_{r}$. This is possible under a certain regularity condition on $w_{r}$ (or $g_{r}$ ), as shown by the following lemma.

Lemma 3.5 Let $w, h, g$ be as in Lemma 3.3. Then the following properties are equivalent:

(i) There exist positive constants $c_{1}, d_{1}$ and $\alpha_{1}$ with $\alpha_{1}>1$ such that

$$
c_{1}\left(\frac{t}{s}\right)^{\alpha_{1}} \leq \frac{w(s)}{w(t)} \quad \text { and } \quad \frac{w(s)}{w(2 s)} \leq d_{1}, \quad \forall t \geq s \geq 1 .
$$

(ii) There exist positive constants $c_{2}, d_{2}$ and $\alpha_{2}$ such that

$$
c_{2}\left(\frac{t}{s}\right)^{\alpha_{2}} \leq \frac{h(s)}{h(t)} \quad \text { and } \quad \frac{h(s)}{h(2 s)} \leq d_{2}, \quad \forall t \geq s \geq 1
$$


(iii) There exist positive constants $c, d$ and $\beta$ with $0<\beta<1$ such that

$$
\frac{g(t)}{g(s)} \leq d\left(\frac{t}{s}\right)^{\beta} \quad \text { and } \quad \frac{g^{-1}(2 s)}{g^{-1}(s)} \leq c, \quad \forall t \geq s \geq 1
$$

Moreover, if one of (i)-(iii) is satisfied, then

$$
c^{\prime} w(t) \leq \frac{1}{g^{-1}(t)} \leq d^{\prime} w(t), \quad \forall t \geq 1,
$$

where the constants $c^{\prime}$ and $d^{\prime}$ depend only on the relative constants in (i)-(iii).

Proof. First note that by (3.3) for $s>0$ and $t=g(s)$ we have

$$
h(t)=\frac{g(s)}{s}=\frac{t}{g^{-1}(t)} .
$$

Using this we easily check (ii) $\Leftrightarrow$ (iii) (with $\beta=\left(1+\alpha_{2}\right)^{-1}$ ) and thus omit the details. Now assume (ii). Let us show (3.9) and (i). We have

$$
w(t) \leq 2 \frac{h(t / 2)}{t} \leq 2 d_{2} \frac{h(t)}{t}, \quad \forall t \geq 2 .
$$

On the other hand, since $w$ is decreasing, $h$ is convex. Thus for any $0<t<s<r$ we have

$$
\frac{h(s)-h(t)}{s-t} \leq \frac{h(r)-h(t)}{r-t} .
$$

Fixing $r$ and letting $s \rightarrow t$, we deduce

$$
-w(t) \leq \frac{h(r)-h(t)}{r-t} .
$$

Now choose $r=\lambda t$ with $c_{2} \lambda^{\alpha_{2}} \geq 2$. Then

$$
h(\lambda t) \leq \frac{h(t)}{c_{2} \lambda^{\alpha_{2}}} \leq \frac{h(t)}{2} .
$$

It then follows that

$$
w(t) \geq \frac{1}{2(\lambda-1)} \frac{h(t)}{t}
$$

Therefore,

$$
w(t) \sim \frac{h(t)}{t}=\frac{1}{g^{-1}(t)} .
$$

This is (3.9) and also yields (i) with $\alpha_{1}=1+\alpha_{2}$. It remains to show (i) $\Rightarrow$ (ii). Assuming (i), we have

$$
h(t) \geq t w(2 t) \geq d_{1}^{-1} t w(t), \quad \forall t \geq 1 .
$$

For the converse inequality we find

$$
h(t) \leq c_{1}^{-1} t^{\alpha_{1}} w(t) \int_{t}^{\infty} s^{-\alpha_{1}} d s=c_{1}^{-1}\left(\alpha_{1}-1\right)^{-1} t w(t) .
$$

Combining this with the previous inequality we get $h(t) \sim t w(t)$. We then deduce (ii) from (i). 
Remark 3.6 The second inequality in (3.6) is satisfied if there exist positive constants $d_{1}$ and $\beta_{1}$ with $\beta_{1}>1$ such that

$$
\frac{w(s)}{w(t)} \leq d_{1}\left(\frac{t}{s}\right)^{\beta_{1}}, \quad \forall t \geq s \geq 1
$$

A similar remark applies to (3.7) and (3.8). However, in the case of (3.8) we can formulate a condition directly on $g$ as follows: there exist positive constants $c$ and $\alpha$ with $0<\alpha<1$ such that

$$
c\left(\frac{y}{x}\right)^{\alpha} \leq \frac{g(y)}{g(x)}, \quad \forall y \geq x \geq 1 .
$$

Remark 3.7 Lemma 3.3 defines the function $g$ starting from $w$. Under the regularity conditions in Lemma 3.5 we are able to reverse this procedure. Namely, let $\mathrm{g}$ be an increasing continuous function on $\mathbb{R}_{+}$satisfying (3.8). Let $w=1 / \mathrm{g}^{-1}$. Let $g$ be the function associated to $w$ by (3.2). Then by Lemma 3.5 $g$ is equivalent to $g$ at infinity: there exist two positive constants $c$ and $d$ such that

$$
c g(x) \leq \mathrm{g}(x) \leq d g(x), \quad \forall x \geq 1 .
$$

We are finally ready to prove our uniqueness theorem on $K_{w_{c}, w_{r}}$.

Theorem 3.8 Let $\mathrm{g}_{c}$ and $\mathrm{g}_{r}$ be two increasing continuous positive functions on $\mathbb{R}_{+}$both satisfying the regularity condition (3.8) for some positive constants $c, d$ and $\beta$ with $0<\beta<1$. Then there exists, up to complete isomorphism, exactly one space $F \in H Q S(C \oplus R)$ such that

$$
\left\|\sum_{k=1}^{n} e_{k 1} \otimes e_{k}\right\|_{C[F]}^{2} \sim \mathrm{g}_{c}(n), \quad\left\|\sum_{k=1}^{n} e_{1 k} \otimes e_{k}\right\|_{R[F]}^{2} \sim \mathrm{g}_{r}(n) \quad \text { uniformly in } n \in \mathbb{N},
$$

where $\left(e_{k}\right)$ is an orthonormal basis of $F$. Moreover, $F$ can be chosen to be $K_{w_{c}, w_{r}}$ with a pair $\left(w_{c}, w_{r}\right)$ of weights on $\mathbb{R}$ satisfying (3.1) and such that

$$
w_{c}(-t) \sim \frac{1}{\mathrm{~g}_{c}^{-1}(t)} \quad \text { and } \quad w_{r}(t) \sim \frac{1}{\mathrm{~g}_{r}^{-1}(t)} \quad \text { for } \quad t \geq 1 .
$$

Proof. By Remarks 3.7 and 3.2 we may perturb $\left(\mathrm{g}_{c}^{-1}, \mathrm{~g}_{r}^{-1}\right)$ into a pair $\left(w_{c}, w_{r}\right)$ of weights on $\mathbb{R}$ verifying (3.1) and (3.6). Therefore, thanks to Lemma 3.4 the space $F=K_{w_{c}, w_{r}}$ satisfies (3.12). Now let $F \in H Q S(C \oplus R)$ satisfy (3.12). Using Theorem 2.3 and Proposition 3.1, we can assume $F=\lambda C \cap \gamma R \cap K_{w_{c}}, w_{r}$. Then we must have $\lambda=\gamma=0$. Indeed, if, for instance, $\lambda>0$ and $\gamma=0$, then $F=C$ and so $\left\|\sum_{k=1}^{n} e_{k 1} \otimes e_{k}\right\|_{C[F]}=\sqrt{n}$, a contradiction with (3.12) and the regularity of $\mathrm{g}_{c}$. Therefore, $F=K_{w_{c}, w_{r}}$. Let $g_{c}$ and $g_{r}$ be the functions associated to $w_{c}$ and $w_{r}$ as in (3.2). Then by Lemma 3.4, $g_{c} \sim \mathrm{g}_{c}$ and $g_{r} \sim \mathrm{g}_{r}$. Therefore, $g_{c}$ and $g_{r}$ satisfy (3.8) too. Thus by (3.9) we deduce

$$
w_{c}(-t) \sim \frac{1}{g_{c}^{-1}(t)} \sim \frac{1}{\mathrm{~g}_{c}^{-1}(t)} \quad \text { and } \quad w_{r}(t) \sim \frac{1}{g_{r}^{-1}(t)} \sim \frac{1}{\mathrm{~g}_{r}^{-1}(t)} \quad \text { for } \quad t \geq 1 .
$$

Hence $F$ is completely isomorphic to the space constructed previously. This shows the uniqueness part.

The preceding results suggest the following

Definition 3.9 Let $F$ be a homogenous Hilbertian operator space. Let

$$
\Phi_{c, F}(n)=\left\|\sum_{k=1}^{n} e_{k 1} \otimes e_{k}\right\|_{C[F]}^{2} \quad \text { and } \quad \Phi_{r, F}(n)=\left\|\sum_{k=1}^{n} e_{1 k} \otimes e_{k}\right\|_{R[F]}^{2}, \quad n \in \mathbb{N}
$$


where $\left(e_{k}\right)$ is a basis of $F$ which is the image of an orthonormal basis under an isomorphism between $F$ and a Hilbert space. We call $\Phi_{c}$ and $\Phi_{r}$ the fundamental functions of $F . F$ is said to be regular if there exist positive constants $c, d$ and $\alpha, \beta$ with $0<\alpha \leq \beta<1$ such that

$$
c\left(\frac{n}{k}\right)^{\alpha} \leq \frac{\Phi_{c, F}(n)}{\Phi_{c, F}(k)} \leq d\left(\frac{n}{k}\right)^{\beta} \quad \text { and } \quad c\left(\frac{n}{k}\right)^{\alpha} \leq \frac{\Phi_{r, F}(n)}{\Phi_{r, F}(k)} \leq d\left(\frac{n}{k}\right)^{\beta}, \quad \forall n \geq k \geq 1 .
$$

We will denote $\Phi_{c, F}$ and $\Phi_{r, F}$ simply by $\Phi_{c}$ and $\Phi_{r}$, respectively whenever no confusion can occur. In the sequel, these functions will be systematically extended to piecewise linear functions on $\mathbb{R}_{+}$with $\Phi_{c}(0)=\Phi_{c}(1)$ and $\Phi_{r}(0)=\Phi_{r}(1)$. In fact, we can even assume, by perturbation, that they are increasing on $\mathbb{R}_{+}$.

Remark 3.10 Note that the homogeneity of $F$ implies

$$
\frac{\Phi_{c}(n)}{\Phi_{c}(k)} \leq d \frac{n}{k} \quad \text { and } \quad \frac{\Phi_{r}(n)}{\Phi_{r}(k)} \leq d \frac{n}{k} .
$$

Thus the regularity of $F$ does not require much more than necessary.

Theorem 3.11 Let $F$ be a 1-homogeneous 1-Hilbertian operator space. Then

$$
\Phi_{c, F}(n) \Phi_{c, F^{*}}(n)=n \quad \text { and } \quad \Phi_{r, F}(n) \Phi_{r, F^{*}}(n)=n, \quad \forall n \in \mathbb{N} .
$$

Proof. By symmetry, it suffices to deal with the column case. Let $n \in \mathbb{N}$. Considering an $n$ dimensional subspace instead of $F$, we can assume $\operatorname{dim} F=n$. We will use the duality equality: $C^{n}[F]^{*}=C_{1}^{n}\left[F^{*}\right]$. Fix an orthonormal basis $\left(e_{k}\right)$ of $F$. We consider, as usual, the elements $x$ in $C^{n}[F]$ or $C_{1}^{n}\left[F^{*}\right]$ as matrices with respect to the bases $\left(e_{k 1}\right)$ and $\left(e_{k}\right)$ :

$$
x=\sum_{i, j=1}^{n} x_{i j} e_{i 1} \otimes e_{j} .
$$

By a slight abuse of notation, we use id to denote the identity matrix as well as the corresponding identity elements in these spaces. Then

$$
\|\operatorname{id}\|_{C^{n}[F]}=\sup \left\{\frac{|\operatorname{Tr}(x)|}{\|x\|_{C_{1}^{n}\left[F^{*}\right]}}: x \in C_{1}^{n}\left[F^{*}\right]\right\},
$$

where $\operatorname{Tr}$ denotes the usual matrix trace. We claim that the supremum above is attained at id. Indeed, let $\mathcal{U}$ denote the group of $n \times n$ unitary matrices, equipped with Haar measure. For any $u \in \mathcal{U}$ and $x \in C_{1}^{n}\left[F^{*}\right]$ we have

$$
\operatorname{Tr}\left(u x u^{*}\right)=\operatorname{Tr}(x) \text { and }\left\|u x u^{*}\right\|_{C_{1}^{n}\left[F^{*}\right]}=\|x\|_{C_{1}^{n}\left[F^{*}\right]},
$$

where the second equality follows from homogeneity. Let

$$
\widetilde{x}=\int_{\mathcal{U}} u x u^{*} d u
$$

Then

$$
\widetilde{x}=\frac{\operatorname{Tr}(x)}{n} \text { id } \quad \text { and } \quad\|\widetilde{x}\|_{C_{1}^{n}\left[F^{*}\right]} \leq\|x\|_{C_{1}^{n}\left[F^{*}\right]} .
$$

Thus the claim follows. Our next task is to calculate $\|\operatorname{id}\|_{C_{1}^{n}\left[F^{*}\right]}$. To this end recall that by $\mathbf{P} 4$, Theorem 1.5] we have

$$
\|\operatorname{id}\|_{C_{1}^{n}\left[F^{*}\right]}=\inf \left\{\|a\|_{S_{2}^{n}}\|y\|_{C^{n}\left[F^{*}\right]}: \operatorname{id}=a y, a \in S_{2}^{n}, y \in C^{n}\left[F^{*}\right]\right\} .
$$


We will show that the infimum is attained at the trivial factorization with $a=$ id and $y=$ id. Let id $=a y$ be a factorization as above. Then $a=y^{-1}$. Let $u, v \in \mathcal{U}$ such that $u y v=\delta$ is a diagonal matrix with positive diagonal entries. By the homogeneity of $F, C^{n}$ and the unitary invariance of $S_{2}^{n}$ we have

$$
\|\delta\|_{C^{n}\left[F^{*}\right]}=\|y\|_{C^{n}\left[F^{*}\right]} \quad \text { and } \quad\left\|\delta^{-1}\right\|_{S_{2}^{n}}=\left\|v^{*} a u^{*}\right\|_{S_{2}^{n}}=\|a\|_{S_{2}^{n}} .
$$

Thus we can replace $y$ by $\delta$ and $a$ by $\delta^{-1}$. Then as before, we have

$$
\int_{\mathcal{U}} u \delta u^{*} d u=\frac{\delta_{1}+\cdots+\delta_{n}}{n} \mathrm{id},
$$

where $\delta_{1}, \ldots, \delta_{n}$ are the diagonal entries of $\delta$. Again by homogeneity, we find

$$
\frac{\delta_{1}+\cdots+\delta_{n}}{n}\|\mathrm{id}\|_{C^{n}\left[F^{*}\right]} \leq\|\delta\|_{C^{n}\left[F^{*}\right]} .
$$

Similarly,

$$
\frac{\delta_{1}^{-1}+\cdots+\delta_{n}^{-1}}{n}\|\mathrm{id}\|_{S_{2}^{n}} \leq\left\|\delta^{-1}\right\|_{S_{2}^{n}} .
$$

We then deduce, by the Cauchy-Schwarz inequality, that

$$
\begin{aligned}
\sqrt{n}\|\mathrm{id}\|_{C^{n}\left[F^{*}\right]} & =\|\mathrm{id}\|_{S_{2}^{n}}\|\mathrm{id}\|_{C^{n}\left[F^{*}\right]} \\
& \leq \frac{\delta_{1}^{-1}+\cdots+\delta_{n}^{-1}}{n} \frac{\delta_{1}+\cdots+\delta_{n}}{n}\|\mathrm{id}\|_{S_{2}^{n}}\|\mathrm{id}\|_{C^{n}\left[F^{*}\right]} \\
& \leq\left\|\delta^{-1}\right\|_{S_{2}^{n}}\|\delta\|_{C^{n}\left[F^{*}\right]} .
\end{aligned}
$$

This yields the announced assertion. Therefore,

$$
\|\mathrm{id}\|_{C_{1}^{n}\left[F^{*}\right]}=\sqrt{n}\|\mathrm{id}\|_{C^{n}\left[F^{*}\right]} .
$$

Combining the previous two parts, we finally get

$$
\|\mathrm{id}\|_{C^{n}[F]}=\frac{\operatorname{Tr}(\mathrm{id})}{\sqrt{n}\|\mathrm{id}\|_{C^{n}\left[F^{*}\right]}}=\frac{\sqrt{n}}{\|\mathrm{id}\|_{C^{n}\left[F^{*}\right]}} .
$$

Recalling that $\Phi_{c, F}=\|\mathrm{id}\|_{C^{n}[F]}^{2}$ and $\Phi_{c, F^{*}}=\|\mathrm{id}\|_{C^{n}\left[F^{*}\right]}^{2}$, we then deduce the theorem.

Corollary 3.12 Let $F$ be a homogeneous Hilbertian space. Then $F$ is regular iff $F^{*}$ is regular.

\section{Completely 1-summing maps}

In this section we deal with completely 1-summing maps between two homogeneous spaces $E$ and $F$ in $Q S(C \oplus R)$. We will show that $\Pi_{1}^{o}(E, F)$ coincides with a Schatten-Orlicz class $S_{\varphi}$. We first recall the relevant definition. Let $\Phi$ be a symmetric sequence space on $\mathbb{N}$ in the sense of [LT]. Recall that the unitary ideal $S_{\Phi}$ is the space of all compact operators $x$ on $\ell_{2}$ such that $\left(s_{n}(x)\right)_{n \geq 1}$ belongs to $\Phi$. Here $\left(s_{n}(x)\right)_{n \geq 1}$ denotes the sequence of singular values of $x$, i.e., $\left(s_{n}(x)\right)_{n \geq 1}$ is the sequence of the eigenvalues of $|x|=\sqrt{x^{*} x}$ ranged in decreasing order and repeated according to multiplicity. $S_{\Phi}$ is equipped with the norm

$$
\|x\|_{\Phi}=\left\|\left(s_{n}(x)\right)_{n \geq 1}\right\|_{\Phi} .
$$

Note that $S_{\Phi}$ is an ideal of $B\left(\ell_{2}\right)$ and its norm is unitarily invariant. Namely, $\|u x\|_{\Phi}=\|x u\|_{\Phi}=$ $\|x\|_{\Phi}$ for any unitary $u \in B\left(\ell_{2}\right)$ and any $x \in S_{\Phi}$. Conversely, any unitarily invariant norm on an ideal of compact operators on $\ell_{2}$ is of this form. We refer to [GK] and [S] for more information. We are particularly interested in the case where $\Phi$ is an Orlicz sequence space $\ell_{\varphi}$. In this case the unitary ideal $S_{\ell_{\varphi}}$ is simply denoted by $S_{\varphi}$. In particular, $S_{\varphi}=S_{p}$ if $\varphi(t)=t^{p}$ with $p<\infty$. Let us adopt the following convention. Let $E$ and $F$ be two (infinite dimensional) homogeneous Hilbertian operator spaces. Identifying both $E$ and $F$ with $\ell_{2}$ isomorphically, we will view maps from $E$ to $F$ as operators on $\ell_{2}$. 
Remark 4.1 Let $E$ and $F$ be two 1-homogenous 1-Hilbertian operator spaces. Then the norm of $\Pi_{1}^{o}(E, F)$ is unitarily invariant. This reduces the determination of the whole space $\Pi_{1}^{o}(E, F)$ to that of the subspace of diagonal operators with respect to two given orthonormal bases in $E$ and $F$, respectively. Note, however, that $\Pi_{1}^{o}(E, F)$ may contain non compact operators in general.

Recall that an Orlicz function $\varphi$ satisfies the $\Delta_{2}$-condition (at 0 ) if there exists a positive constant $\lambda$ such that $\varphi(2 t) \leq \lambda \varphi(t)$ for all $t>0$ (in a neighborhood of 0 ). It is well-known that $\varphi$ satisfies the $\Delta_{2}$-condition at 0 iff the finite sequences are dense in $\ell_{\varphi}$ (see [LT]).

Theorem 4.2 Let $E, F \in H Q S(C \oplus R)$ be infinite dimensional. Then $\Pi_{1}^{o}(E, F) \subset S_{2}$ and there exists an Orlicz function $\varphi$ satisfying the $\Delta_{2}$-condition such that $\Pi_{1}^{o}(E, F)=S_{\varphi}$ isomorphically. Moreover, all relevant constants depend only on the homogeneity constants of $E$ and $F$.

We require the following lemma for the proof. Let us recall that the noncommutative $L_{1}(\mathcal{M})$ associated with a von Neumann algebra $\mathcal{M}$ is equipped with its natural operator space structure. Namely, as operator space, $L_{1}(\mathcal{M})$ is the predual of the opposite von Neumann algebra $\mathcal{M}^{\text {op }}$ of $\mathcal{M}$ (see [P5, Chapiter 7]). Let $E$ and $F$ be two subspaces of $L_{1}(\mathcal{M})$. We denote by $E \otimes_{1} F$ the closure of $E \otimes F$ in $L_{1}(\mathcal{M} \otimes \mathcal{M})$. As usual, we identify an element of $E \otimes F$ with its associated map from $E^{*}$ to $F$. Recall that a von Neumann algebra is called QWEP if it is a quotient of a von Neumann algebra with the WEP (weak expectation property).

Lemma 4.3 Let $\mathcal{M}$ be a $Q W E P$ von Neumann algebra and let $E, F \subset L_{1}(\mathcal{M})$ be finite dimensional subspaces. Then $\Pi_{1}^{o}\left(E^{*}, F\right)=E \otimes_{1} F$ isometrically.

This is $\mathrm{Y}$, Corollary 10]. It is also implicitly contained in [J1. Note that we will need this lemma only for injective $\mathcal{M}$. In this latter case, it immediately follows from [P4, Lemma 5.14].

Proof of Theorem 4.2, By Corollary 2.7, we have

$$
C \cap R \subset E, F \subset C+R .
$$

Consequently, $\Pi_{1}^{o}(E, F) \subset \Pi_{1}^{o}(C \cap R, C+R)$. However, it is easy to check that $\Pi_{1}^{o}(C \cap R, C+R)$ coincides with $S_{2}$ isometrically (see case 4 below). Thus $\Pi_{1}^{o}(E, F) \subset S_{2}$. Therefore, by Remark 4.1. $\Pi_{1}^{o}(E, F)=S_{\Phi}$ for some symmetric sequence space $\Phi$. The main part of the proof is, of course, to show that $\Phi$ is an Orlicz sequence space.

By Corollary 2.7 once more, $E^{*}$ and $F$ are completely isomorphic to $C, R, C \cap R$ or spaces of the form $K_{u_{c}, u_{r}}$ for $E^{*}$ belongs to $H Q S(C \oplus R)$ too. We will consider different cases according to the isomorphism types of $E^{*}$ and $F$. The most important one is where both $E^{*}$ and $F$ are of type $K_{u_{c}, u_{r}}$.

Case 1: $E^{*}=K_{u_{c}, u_{r}}$ and $F=K_{v_{c}, v_{r}}$ for two pairs of weights on $(\Omega, \nu)$. We will denote by $\left(e_{k}\right)$ the canonical basis of both $K_{u_{c}, u_{r}}$ and $K_{v_{c}, v_{r}}$ (so $E^{*}$ and $F$ are identified with $\ell_{2}$ at the Banach space level). By [X], $G_{u_{c}, u_{r}}$ and $G_{v_{c}, v_{r}}$ completely embed into a noncommutative $L_{1}(\mathcal{M})$ with $\mathcal{M}$ a QWEP von Neumann algebra with universal constants. We point out that $\mathcal{M}$ can be further chosen to be injective by virtue of [J2] (see also [HM1] for a different approach). Without loss of generality assume that all these spaces are subspaces of $L_{1}(\mathcal{M})$. Then using Lemma 4.3 we deduce

$$
\Pi_{1}^{o}(E, F)=E^{*} \otimes_{1} F \subset G_{u_{c}, u_{r}} \otimes_{1} G_{v_{c}, v_{r}} .
$$

Indeed, since $E$ and $F$ are homogeneous and Hilbertian, we can easily reduce the equality above to the finite dimensional case. Assume further that all column and row spaces considered below are subspaces of $L_{1}(\mathcal{M})$. By the definition of $G_{u_{c}, u_{r}}$, we have

$$
\begin{aligned}
G_{u_{c}, u_{r}} \otimes_{1} G_{v_{c}, v_{r}}= & L_{2}^{c}\left(u_{c} ; \ell_{2}\right) \otimes_{1} L_{2}^{c}\left(v_{c} ; \ell_{2}\right)+L_{2}^{r}\left(u_{r} ; \ell_{2}\right) \otimes_{1} L_{2}^{r}\left(v_{r} ; \ell_{2}\right)+ \\
& L_{2}^{c}\left(u_{c} ; \ell_{2}\right) \otimes_{1} L_{2}^{r}\left(v_{r} ; \ell_{2}\right)+L_{2}^{r}\left(u_{r} ; \ell_{2}\right) \otimes_{1} L_{2}^{c}\left(v_{c} ; \ell_{2}\right) .
\end{aligned}
$$


Note that for two Hilbert spaces $H$ and $K$

$$
H^{c} \otimes_{1} K^{c}=\left(H \otimes_{2} K\right)^{c} \quad \text { and } \quad H^{c} \otimes_{1} K^{r}=H^{c} \widehat{\otimes} K^{r}=S_{1}\left(H^{*}, K\right)
$$

hold completely isometrically, where $\otimes_{2}$ denotes the Hilbert space tensor product. Recall that $S_{1}\left(H^{*}, K\right)$ is the trace class of operators from $H^{*}$ to $K$. Thus

$$
L_{2}^{c}\left(u_{c} ; \ell_{2}\right) \otimes_{1} L_{2}^{c}\left(v_{c} ; \ell_{2}\right)=L_{2}^{c}\left(u_{c} \otimes v_{c} ; \ell_{2}\left(\mathbb{N}^{2}\right)\right)=L_{2}^{c}\left(u_{c} \otimes v_{c} ; S_{2}\right),
$$

where $u_{c} \otimes v_{c}$ denotes the tensor product weight of $u_{c}$ and $v_{c}$ on $(\Omega \times \Omega, \nu \otimes \nu)$. Similarly,

$$
L_{2}^{r}\left(u_{r} ; \ell_{2}\right) \otimes_{1} L_{2}^{r}\left(v_{r} ; \ell_{2}\right)=L_{2}^{r}\left(u_{r} \otimes v_{r} ; S_{2}\right) .
$$

On the other hand,

$$
L_{2}^{c}\left(u_{c} ; \ell_{2}\right) \otimes_{1} L_{2}^{r}\left(v_{r} ; \ell_{2}\right)=L_{2}^{c}\left(u_{c}\right) \widehat{\otimes} L_{2}^{r}\left(v_{r}\right) \widehat{\otimes} S_{1}
$$

and a similar formula for $L_{2}^{r}\left(u_{r} ; \ell_{2}\right) \otimes_{1} L_{2}^{c}\left(v_{c} ; \ell_{2}\right)$. Combining the preceding equalities we deduce

$$
\begin{aligned}
G_{u_{c}, u_{r}} \otimes_{1} G_{v_{c}, v_{r}}= & L_{2}^{c}\left(u_{c} \otimes v_{c} ; S_{2}\right)+L_{2}^{r}\left(u_{r} \otimes v_{r} ; S_{2}\right)+ \\
& \left(L_{2}^{c}\left(u_{c}\right) \widehat{\otimes} L_{2}^{r}\left(v_{r}\right)+L_{2}^{r}\left(u_{r}\right) \widehat{\otimes} L_{2}^{c}\left(v_{c}\right)\right) \widehat{\otimes} S_{1}
\end{aligned}
$$

completely isomorphically. We now consider these spaces only at the Banach space level. It is easy to check that

$$
L_{2}\left(u_{c} \otimes v_{c} ; X\right)+L_{2}\left(u_{r} \otimes v_{r} ; X\right)=L_{2}\left(\min \left(u_{c} \otimes v_{c}, u_{r} \otimes v_{r}\right) ; X\right)
$$

holds for any Banach space $X$ with universal equivalence constants. Consequently,

$$
L_{2}^{c}\left(u_{c} \otimes v_{c} ; S_{2}\right)+L_{2}^{r}\left(u_{r} \otimes v_{r} ; S_{2}\right)=A \otimes_{2} S_{2},
$$

where

$$
A=L_{2}\left(\min \left(u_{c} \otimes v_{c}, u_{r} \otimes v_{r}\right)\right) .
$$

Let $\otimes_{\pi}$ denote the Banach space projective tensor product. We have

$$
L_{2}^{c}\left(u_{c}\right) \widehat{\otimes} L_{2}^{r}\left(v_{r}\right)+L_{2}^{r}\left(u_{r}\right) \widehat{\otimes} L_{2}^{c}\left(v_{c}\right)=L_{2}\left(u_{c}\right) \otimes_{\pi} L_{2}\left(v_{r}\right)+L_{2}\left(u_{r}\right) \otimes_{\pi} L_{2}\left(v_{c}\right) \stackrel{\text { def }}{=} B .
$$

Now let $x \in \Pi_{1}^{o}(E, F)$. By Remark 4.1, we can assume that $x$ is a diagonal operator:

$$
x=\sum_{k} x_{k} e_{k} \otimes e_{k} .
$$

Then by (4.1) we deduce that

$$
\pi_{1}^{o}(x) \sim_{c} \inf \left\{\|y\|_{L_{2}^{c}\left(u_{c} \otimes v_{c} ; S_{2}\right)+L_{2}^{r}\left(u_{r} \otimes v_{r} ; S_{2}\right)}+\|z\|_{\left(L_{2}^{c}\left(u_{c}\right) \widehat{\otimes} L_{2}^{r}\left(v_{r}\right)+L_{2}^{r}\left(u_{r}\right) \widehat{\otimes} L_{2}^{c}\left(v_{c}\right)\right) \widehat{\otimes} S_{1}}\right\},
$$

where the infimum runs over all decompositions $x_{k}=y_{k k}(\omega, \sigma)+z_{k k}(\omega, \sigma)$ for almost all $(\omega, \sigma) \in$ $\Omega \times \Omega$ and all $k \in \mathbb{N}$ with

$$
\begin{aligned}
& y=\left(y_{k l}\right) \in L_{2}^{c}\left(u_{c} \otimes v_{c} ; S_{2}\right)+L_{2}^{r}\left(u_{r} \otimes v_{r} ; S_{2}\right), \\
& z=\left(z_{k l}\right) \in\left(L_{2}^{c}\left(u_{c}\right) \widehat{\otimes} L_{2}^{r}\left(v_{r}\right)+L_{2}^{r}\left(u_{r}\right) \widehat{\otimes} L_{2}^{c}\left(v_{c}\right)\right) \widehat{\otimes} S_{1} .
\end{aligned}
$$

Here we have viewed the elements in $L_{2}^{c}\left(u_{c} \otimes v_{c} ; S_{2}\right)+L_{2}^{r}\left(u_{r} \otimes v_{r} ; S_{2}\right)$ as matrices with entries in $L_{2}^{c}\left(u_{c} \otimes v_{c}\right)+L_{2}^{r}\left(u_{r} \otimes v_{r}\right)$ and similarly for the other space. Note that the diagonal projection on $S_{2}$ extends to contractive projections on both $L_{2}^{c}\left(u_{c} \otimes v_{c} ; S_{2}\right)+L_{2}^{r}\left(u_{r} \otimes v_{r} ; S_{2}\right)$ and 
$\left(L_{2}^{c}\left(u_{c}\right) \widehat{\otimes} L_{2}^{r}\left(v_{r}\right)+L_{2}^{r}\left(u_{r}\right) \widehat{\otimes} L_{2}^{c}\left(v_{c}\right)\right) \widehat{\otimes} S_{1}$. Therefore, the infimum in (4.4) can be restricted to all decompositions with diagonal $y$ and $z$. However, for a diagonal $y$ using (4.2) we find

$$
\|y\|_{L_{2}^{c}\left(u_{c} \otimes v_{c} ; S_{2}\right)+L_{2}^{r}\left(u_{r} \otimes v_{r} ; S_{2}\right)} \sim\|y\|_{\left.\ell_{2}(A)\right)}=\left(\sum_{k}\left\|y_{k}\right\|_{A}^{2}\right)^{1 / 2} .
$$

On the other hand, if $z$ is diagonal, we have, by virtue of (4.3)

$$
\begin{aligned}
\|z\|_{\left(L_{2}^{c}\left(u_{c}\right) \widehat{\otimes} L_{2}^{r}\left(v_{r}\right)+L_{2}^{r}\left(u_{r}\right) \widehat{\otimes} L_{2}^{c}\left(v_{c}\right)\right) \widehat{\otimes} S_{1}} & =\|z\|_{\left(L_{2}^{c}\left(u_{c}\right) \widehat{\otimes} L_{2}^{r}\left(v_{r}\right)+L_{2}^{r}\left(u_{r}\right) \widehat{\otimes} L_{2}^{c}\left(v_{c}\right)\right) \widehat{\otimes} \ell_{1}} \\
& =\|z\|_{\ell_{1}\left(L_{2}^{c}\left(u_{c}\right) \widehat{\otimes} L_{2}^{r}\left(v_{r}\right)+L_{2}^{r}\left(u_{r}\right) \widehat{\otimes} L_{2}^{c}\left(v_{c}\right)\right)} \\
& =\|z\|_{\ell_{1}(B)} .
\end{aligned}
$$

It thus follows that

$$
\pi_{1}^{o}(x) \sim_{c} \inf \left\{\left(\sum_{k}\left\|y_{k}\right\|_{A}^{2}\right)^{1 / 2}+\sum_{k}\left\|z_{k}\right\|_{B}\right\}
$$

where the infimum runs over all decompositions $x_{k}=y_{k}(\omega, \sigma)+z_{k}(\omega, \sigma)$ for almost all $(\omega, \sigma) \in \Omega \times \Omega$ and all $k \in \mathbb{N}$ with $y_{k} \in A$ and $z_{k} \in B$. This is the key formula of the proof. It suggests the definition of the desired Orlicz function $\varphi$. Indeed, define $\widetilde{\varphi}: \mathbb{R}_{+} \rightarrow \mathbb{R}_{+}$by

$$
\widetilde{\varphi}(t)=\inf \left\{t^{2}\|a\|_{A}^{2}+t\|b\|_{B}: a \in A, b \in B \text { s.t. } a+b=1 \text { a.e. on } \Omega \times \Omega\right\} .
$$

Note that $\widetilde{\varphi}$ satisfies the following properties:

(i) $\widetilde{\varphi}$ is continuous, $\widetilde{\varphi}(0)=0$ and $\lim _{t \rightarrow \infty} \widetilde{\varphi}(t)=\infty$;

(ii) both $\widetilde{\varphi}$ and $\widetilde{\varphi} / t$ are nondecreasing;

(iii) $\widetilde{\varphi}(2 t) \leq 4 \widetilde{\varphi}(t)$, so $\widetilde{\varphi}$ verifies the $\Delta_{2}$-condition.

Let

$$
\varphi(t)=\int_{0}^{t} \frac{\widetilde{\varphi}(s)}{s} d s .
$$

Then $\varphi$ is an Orlicz function satisfying the $\Delta_{2}$-condition. Moreover,

$$
\varphi(t) \leq \widetilde{\varphi}(t) \leq 4 \varphi(t), \quad \forall t \in \mathbb{R}_{+} .
$$

Therefore $\varphi$ is equivalent to $\widetilde{\varphi}$.

It is now easy to show that $\pi_{1}^{o}(x) \sim_{c}\|x\|_{\varphi}$ for any $x=\left(x_{k}\right)$. Assume $\|x\|_{\varphi}<1$. Then there exist $\left(a_{k}\right)$ and $\left(b_{k}\right)$ such that $a_{k}+b_{k}=1$ a.e. on $\Omega \times \Omega$ and

$$
\sum_{k}\left(\left|x_{k}\right|^{2}\left\|a_{k}\right\|_{A}^{2}+\left|x_{k}\right|\left\|b_{k}\right\|_{B}\right) \leq 4 .
$$

Set $y_{k}=x_{k} a_{k}$ and $z_{k}=x_{k} b_{k}$. Then $x_{k}=y_{k}+z_{k}$ a.e. on $\Omega \times \Omega$ and

$$
\sum_{k}\left\|y_{k}\right\|_{A}^{2}+\sum_{k}\left\|z_{k}\right\|_{B} \leq 4
$$

Thus by (4.5), $\pi_{1}^{o}(x) \leq c$. Conversely, let $x$ be such that the infimum in (4.5) is less than 1 . Choose two sequences $\left(y_{k}\right)$ and $\left(z_{k}\right)$ such that $x_{k}=y_{k}+z_{k}$ and

$$
\left(\sum_{k}\left\|y_{k}\right\|_{A}^{2}\right)^{1 / 2}+\sum_{k}\left\|z_{k}\right\|_{B}<1 .
$$


Then

$$
\sum_{k}\left(\left|x_{k}\right|^{2}\left\|a_{k}\right\|_{A}^{2}+\left|u_{k}\right|\left\|b_{k}\right\|_{B}\right)<1
$$

where $a_{k}=y_{k} / x_{k}$ and $b_{k}=z_{k} / x_{k}$. Since $a_{k}+b_{k}=1$, we find

$$
\sum_{k} \varphi\left(\left|x_{k}\right|\right) \leq \sum_{k} \widetilde{\varphi}\left(\left|x_{k}\right|\right)<1
$$

whence $\|x\|_{\varphi} \leq 1$. Therefore, we get the desired equivalence $\pi_{1}^{o}(x) \sim_{c}\|x\|_{\varphi}$. This shows the theorem in the case where $E^{*}=K_{u_{c}, u_{r}}$ and $F=K_{v_{c}, v_{r}}$.

Case 2: $E^{*} \in\{C, R, C \cap R\}$ and $F=K_{v_{c}, v_{r}}$. The proof in this case is similar to the previous one but much simpler. Consider first the case where $E^{*}=C$. Using the identification $C \simeq R_{1}$, we have

$$
C \otimes_{1} G_{v_{c}, v_{r}}=R_{1}\left[G_{v_{c}, v_{r}}\right]=R_{1}\left[L_{2}^{c}\left(v_{c} ; \ell_{2}\right)\right]+R_{1}\left[L_{2}^{r}\left(v_{r} ; \ell_{2}\right)\right]=L_{2}^{c}\left(v_{c} ; S_{2}\right)+L_{2}^{r}\left(v_{r}\right) \widehat{\otimes} S_{1} .
$$

This is the analogue of (4.1) for the present case. Then as before, for a diagonal operator $x \in$ $\Pi_{1}^{o}(E, F)$ we have

$$
\pi_{1}^{o}(x) \sim_{c} \inf \left\{\left(\sum_{k}\left\|y_{k}\right\|_{L_{2}\left(v_{c}\right)}^{2}\right)^{1 / 2}+\sum_{k}\left\|z_{k}\right\|_{L_{2}\left(v_{r}\right)}\right\}
$$

where the infimum runs over all decompositions $x_{k}=y_{k}(\omega)+z_{k}(\omega)$ for almost all $\omega \in \Omega$ and $k \in \mathbb{N}$ with $\left(y_{k}\right) \subset L_{2}\left(v_{c}\right)$ and $\left(z_{k}\right) \subset L_{2}\left(v_{r}\right)$. Like in case 1 , we deduce that $\pi_{1}^{o}(x) \sim_{c}\|x\|_{\varphi}$, where $\varphi$ is an Orlicz function equivalent to

$$
\widetilde{\varphi}(t)=\inf \left\{t^{2}\|a\|_{L_{2}\left(v_{c}\right)}^{2}+t\|b\|_{L_{2}\left(v_{r}\right)}: a \in L_{2}\left(v_{c}\right), b \in L_{2}\left(v_{r}\right) \text { s.t. } a+b=1 \text { a.e. on } \Omega\right\} .
$$

The case $E^{*}=R$ is dealt with similarly. Now assume $E^{*}=C \cap R$. Then

$$
(C \cap R) \otimes_{1} G_{v_{c}, v_{r}}=\left(L_{2}^{c}\left(v_{c} ; S_{2}\right)+L_{2}^{r}\left(v_{r}\right) \widehat{\otimes} S_{1}\right) \cap\left(L_{2}^{c}\left(v_{c}\right) \widehat{\otimes} S_{1}+L_{2}^{r}\left(v_{r} ; S_{2}\right)\right) .
$$

Therefore for a diagonal operator $x \in \Pi_{1}^{o}(E, F)$ we deduce

$$
\pi_{1}^{o}(x) \sim_{c} \inf \left\{\left[\sum_{k}\left(\left\|y_{k}\right\|_{L_{2}\left(v_{c}\right)}^{2}+\left\|z_{k}^{\prime}\right\|_{L_{2}\left(v_{r}\right)}^{2}\right)\right]^{1 / 2}+\sum_{k}\left(\left\|y_{k}^{\prime}\right\|_{L_{2}\left(v_{c}\right)}+\left\|z_{k}\right\|_{L_{2}\left(v_{r}\right)}\right)\right\}
$$

where the infimum runs over all decompositions $x_{k}=y_{k}+z_{k}=y_{k}^{\prime}+z_{k}^{\prime}$ a.e. on $\Omega$ for $k \in \mathbb{N}$ with $\left(y_{k}\right),\left(y_{k}^{\prime}\right) \subset L_{2}\left(v_{c}\right)$ and $\left(z_{k}\right),\left(z_{k}^{\prime}\right) \subset L_{2}\left(v_{r}\right)$. This time, the required Orlicz function $\varphi$ is equivalent to the following one

$$
\begin{array}{r}
\tilde{\varphi}(t)=\inf \left\{t^{2}\left(\|a\|_{L_{2}\left(v_{c}\right)}^{2}+\left\|b^{\prime}\right\|_{L_{2}\left(v_{r}\right)}^{2}\right)+t\left(\left\|a^{\prime}\right\|_{L_{2}\left(v_{c}\right)}+\|b\|_{L_{2}\left(v_{r}\right)}\right):\right. \\
\left.a, a^{\prime} \in L_{2}\left(v_{c}\right), b, b^{\prime} \in L_{2}\left(v_{r}\right) \text { s.t. } a+b=1=a^{\prime}+b^{\prime}\right\} .
\end{array}
$$

Case 3: $E^{*}=K_{u_{c}, u_{r}}$ and $F \in\{C, R, C \cap R\}$. This is symmetric to case 2 and is proved in the same way. We thus omit the details.

Case 4: $E^{*}, F \in\{C, R, C \cap R\}$. This is the trivial case since we have the following easily checked isometric identifications

$$
\begin{aligned}
& \Pi_{1}^{o}(C, C)=\Pi_{1}^{o}(R, R)=S_{1}, \quad \Pi_{1}^{o}(C, R)=\Pi_{1}^{o}(C, R)=S_{2}, \\
& \Pi_{1}^{o}(C+R, C)=\Pi_{1}^{o}(C+R, R)=\Pi_{1}^{o}(C+R, C \cap R)=S_{1} .
\end{aligned}
$$

Let us check, for instance, $\Pi_{1}^{o}(C+R, C)=S_{2}$. We have

$$
\Pi_{1}^{o}(C+R, C)=(C \cap R) \otimes_{1} C=\left(R_{1} \cap C_{1}\right) \otimes_{1} R_{1}=R_{1}\left[R_{1}\right] \cap C_{1}\left[R_{1}\right]=S_{2} \cap S_{1}=S_{1} .
$$


Therefore, the proof of the theorem is complete.

Since an Orlicz function $\varphi$ is uniquely determined by its fundamental sequence $\left(\varphi_{n}\right)_{n \geq 1}$

$$
\varphi_{n}=\left\|\sum_{k \leq n} e_{k}\right\|_{\varphi}=\frac{1}{\varphi^{-1}\left(\frac{1}{n}\right)}
$$

Theorem 4.2 reduces the calculation of the space $\Pi_{1}^{o}(E, F)$ to that of $\pi_{1}^{o}\left(\mathrm{id}_{n}: E_{n} \rightarrow F_{n}\right)$ for all $n$, where $E_{n}$ and $F_{n}$ are the linear spans of $\left\{e_{1}, \ldots, e_{n}\right\}$ in $E$ and $F$, respectively, and where $\operatorname{id}_{n}: E_{n} \rightarrow F_{n}$ denotes the formal identity from $E_{n}$ to $F_{n}$, i.e., $\operatorname{id}_{n}\left(e_{k}\right)=e_{k}$ for every $k=1, \ldots, n$. We record this in the following

Corollary 4.4 With the notations above $\Pi_{1}^{o}(E, F)$ is uniquely determined, up to isomorphism, by the sequence $\left(\pi_{1}^{o}\left(\operatorname{id}_{n}: E_{n} \rightarrow F_{n}\right)\right)_{n \geq 1}$.

This result considerably simplifies the task of determining $\Pi_{1}^{o}(E, F)$. Thanks to Theorem 4.2 , the only things left to be calculated are the completely 1-summing norms of the identities. For these we have a more concrete formula:

Proposition 4.5 Let $\left(u_{c}, u_{r}\right)$ and $\left(v_{c}, v_{r}\right)$ be two pairs of weights on $(\Omega, \nu)$ satisfying (2.1). Let $E^{*}=K_{u_{c}, u_{r}}$ and $F=K_{v_{c}, v_{r}}$. Then

$$
\pi_{1}^{o}\left(\operatorname{id}_{n}\right) \sim_{c} \inf \left\{\sqrt{n}\|a\|_{A}+n\|b\|_{B}: a \in A, b \in B, a+b=1 \text { a.e. on } \Omega\right\} .
$$

where

$$
A=L_{2}\left(\min \left(u_{c} \otimes v_{c}, u_{r} \otimes v_{r}\right)\right), \quad B=L_{2}\left(u_{c}\right) \otimes_{\pi} L_{2}\left(v_{r}\right)+L_{2}\left(u_{r}\right) \otimes_{\pi} L_{2}\left(v_{c}\right) .
$$

Proof. Consider the projection $P_{n}$ defined for sequences $x=\left(x_{k}\right)$ by

$$
P_{n}(x)=\frac{x_{1}+\cdots+x_{n}}{n} \sum_{k=1}^{n} e_{k} .
$$

Then $P_{n}$ extends to a contractive projection on both $\ell_{2}(A)$ and $\ell_{1}(B)$. Together with (4.5), this implies (4.6).

The remainder of this section is essentially devoted to the proof of Theorem 0.4

Proof of Theorem 0.4. The first part of the theorem is already contained in Theorem 4.2. We will prove the estimate on the fundamental sequence of $\varphi$. Recall that the fundamental functions $\Phi_{c, E^{*}}, \Phi_{r, E^{*}}, \Phi_{c, F}$ and $\Phi_{r, F}$ are extended to continuous functions on $\mathbb{R}_{+}$. Without loss of generality wa can further assume that they are increasing on $\mathbb{R}_{+}$and equal 1 at 0 . Let

$$
w_{c, F}(t)=\left\{\begin{array}{ll}
1 & \text { if } t>0 \\
\frac{1}{\Phi_{c, F}^{-1}(-t)} & \text { if } t \leq 0
\end{array} \quad \text { and } \quad w_{r, F}(t)=\left\{\begin{array}{ll}
1 & \text { if } t<0 \\
\frac{1}{\Phi_{r, F}^{-1}(t)} & \text { if } t \geq 0
\end{array} .\right.\right.
$$

The pair $\left(w_{c, E^{*}}, w_{r, E^{*}}\right)$ has the same meaning with $E^{*}$ in place of $F$. Then by Theorem 4.2

$$
E^{*}=K_{w_{c, E^{*}}, w_{r, E^{*}}} \quad \text { and } \quad F=K_{w_{c, F}, w_{r, F}} .
$$

Now we are in a position to apply (4.6). According to the four quadrants of the plane, we decompose the space $A$ there as follows

$$
\begin{aligned}
A & =L_{2}\left(\min \left(w_{c, E^{*}}^{-} \otimes w_{c, F}^{-}, w_{r, E^{*}}^{-} \otimes w_{r, F}^{-}\right)\right) \oplus L_{2}\left(\min \left(w_{c, E^{*}}^{+} \otimes w_{c, F}^{+}, w_{r, E^{*}}^{+} \otimes w_{r, F}^{+}\right)\right) \\
& \oplus L_{2}\left(\min \left(w_{c, E^{*}}^{-} \otimes w_{c, F}^{+}, w_{r, E^{*}}^{-} \otimes w_{r, F}^{+}\right)\right) \oplus L_{2}\left(\min \left(w_{c, E^{*}}^{+} \otimes w_{c, F}^{-}, w_{r, E^{*}}^{+} \otimes w_{r, F}^{-}\right)\right) \\
& \stackrel{\text { def }}{=} A_{--} \oplus A_{++} \oplus A_{-+} \oplus A_{+--},
\end{aligned}
$$


where

$$
w^{-}=\left.w\right|_{\mathbb{R}_{-}} \quad \text { and } \quad w^{+}=\left.w\right|_{\mathbb{R}_{+}} .
$$

A similar decomposition holds for $B$. Consequently, the infimum in (4.6) is a sum of four infima corresponding to these decompositions. More precisely, we have

$$
\pi_{1}^{o}\left(\mathrm{id}_{n}\right) \sim\|1\|_{\sqrt{n} A_{--}+n B_{--}}+\|1\|_{\sqrt{n} A_{++}+n B_{++}}+\|1\|_{\sqrt{n} A_{-+}+n B_{-+}}+\|1\|_{\sqrt{n} A_{+-}+n B_{+-}} .
$$

Here and during this proof all equivalence constants depend only on the homogeneity and regularity constants of $E$ and $F$. Thus it suffices to deal with separately the four terms on the right. The first two are easy. Indeed, we have

$$
A_{--}=L_{2}\left(\min \left(w_{c, E^{*}}^{-} \otimes w_{c, F}^{-}, 1 \otimes 1\right)\right)=L_{2}\left(w_{c, E^{*}}^{-} \otimes w_{c, F}^{-}\right) .
$$

Since $w_{c, E^{*}}^{-} \otimes w_{c, F}^{-}$is integrable on $\mathbb{R}_{-} \times \mathbb{R}_{-}, 1 \in L_{2}\left(w_{c, E^{*}}^{-} \otimes w_{c, F}^{-}\right)$. Thus

$$
\|1\|_{\sqrt{n} A_{--}+n B_{--}} \sim \sqrt{n} .
$$

Similarly,

$$
\|1\|_{\sqrt{n} A_{++}+n B_{++}} \sim \sqrt{n} .
$$

Therefore,

$$
\pi_{1}^{o}\left(\mathrm{id}_{n}\right) \sim\|1\|_{\sqrt{n} A_{-+}+n B_{-+}}+\|1\|_{\sqrt{n} A_{+-}+n B_{+-}} .
$$

To estimate the last two terms, note that

$$
A_{-+}=L_{2}\left(\min \left(w_{c, E^{*}}^{-} \otimes 1,1 \otimes w_{r, F}^{+}\right)\right)
$$

and

$$
B_{-+}=L_{2}\left(w_{c, E^{*}}^{-}\right) \otimes_{\pi} L_{2}\left(w_{r, F}^{+}\right)+L_{2}\left(\mathbb{R}_{-}\right) \otimes_{\pi} L_{2}\left(\mathbb{R}_{+}\right)=L_{2}\left(w_{c, E^{*}}^{-}\right) \otimes_{\pi} L_{2}\left(w_{r, F}^{+}\right) .
$$

Since the projective tensor norm dominates the Hilbert tensor norm, we have the following contractive inclusion

$$
B_{-+} \subset L_{2}\left(w_{c, E^{*}}^{-}\right) \otimes_{2} L_{2}\left(w_{r, F}^{+}\right)=L_{2}\left(w_{c, E^{*}}^{-} \otimes w_{r, F}^{+}\right) .
$$

Hence,

$$
\|1\|_{\sqrt{n} A_{-+}+n B_{-+}} \geq c^{-1}\|1\|_{L_{2}\left(\min \left(n w_{c, E^{*}}^{-} \otimes 1, n 1 \otimes w_{r, F}^{+}, n^{2} w_{c, E^{*}}^{-} \otimes w_{r, F}^{+}\right)\right)} .
$$

To ease the calculation of the minimum above we set $\widetilde{w}_{c, E^{*}}(s)=w_{c, E^{*}}^{-}(-s)$ for $s \in \mathbb{R}_{+}$. Since $\widetilde{w}_{c, E^{*}}$ and $w_{r, F}^{+}$are decreasing on $\mathbb{R}_{+}$, for each $s \in \mathbb{R}_{+}$there exists a unique $t=t(s) \in \mathbb{R}_{+}$such that

$$
\widetilde{w}_{c, E^{*}}(s)=w_{r, F}^{+}(t(s)) .
$$

The function $s \mapsto t(s)$ is increasing and bijective on $\mathbb{R}_{+}$. Its inverse is the function $t \mapsto s(t)$ such that

$$
\widetilde{w}_{c, E^{*}}(s(t))=w_{r, F}^{+}(t) .
$$

We then have

$$
\min \left(\widetilde{w}_{c, E^{*}}(s), w_{r, F}^{+}(t)\right)= \begin{cases}\widetilde{w}_{c, E^{*}}(s) & \text { if } t \leq t(s), \\ w_{r, F}^{+}(t) & \text { if } t>t(s) .\end{cases}
$$

On the other hand, for each $n \in \mathbb{N}$ there exist (unique) $s_{n}, t_{n} \in \mathbb{R}_{+}$such that

$$
\widetilde{w}_{c, E^{*}}\left(s_{n}\right)=w_{r, F}^{+}\left(t_{n}\right)=\frac{1}{n} .
$$


Note that $t_{n}=t\left(s_{n}\right)$. We now decompose $\mathbb{R}_{+} \times \mathbb{R}_{+}$into a union of three disjoint regions:

$$
\begin{aligned}
& \Lambda_{1}=\left\{(s, t): t \leq t_{n}, s \geq s(t)\right\}, \\
& \Lambda_{2}=\left\{(s, t): s \leq s_{n}, t>t(s)\right\}, \\
& \Lambda_{3}=\left(s_{n}, \infty\right) \times\left(t_{n}, \infty\right) .
\end{aligned}
$$

Then

$$
\min \left(n \widetilde{w}_{c, E^{*}}(s), n w_{r, F}^{+}(t), n^{2} \widetilde{w}_{c, E^{*}}(s) w_{r, F}^{+}(t)\right)= \begin{cases}n \widetilde{w}_{c, E^{*}}(s) & \text { if }(s, t) \in \Lambda_{1}, \\ n w_{r, F}^{+}(t) & \text { if }(s, t) \in \Lambda_{2}, \\ n^{2} \widetilde{w}_{c, E^{*}}(s) w_{r, F}^{+}(t) & \text { if }(s, t) \in \Lambda_{3} .\end{cases}
$$

Therefore,

$$
\begin{aligned}
& \|1\|_{L_{2}\left(\min \left(n w_{c, E^{*}}^{-} \otimes 1, n 1 \otimes w_{r, F}^{+}, n^{2} w_{c, E^{*}}^{-} \otimes w_{r, F}^{+}\right)\right)}^{2} \\
& \quad=n \int_{\Lambda_{1}} \widetilde{w}_{c, E^{*}}(s) d s d t+n \int_{\Lambda_{2}} w_{r, F}^{+}(t) d s d t+n^{2} \int_{\Lambda_{3}} \widetilde{w}_{c, E^{*}}(s) w_{r, F}^{+}(t) d s d t \\
& \stackrel{\text { def }}{=} \lambda_{1}+\lambda_{2}+\lambda_{3} .
\end{aligned}
$$

Recall that $h_{c, E^{*}}$ and $g_{c, E^{*}}$ are the functions associated to $\widetilde{w}_{c, E^{*}}$ by (3.2). Then by (3.3)

$$
\begin{aligned}
\frac{\lambda_{3}}{n^{2}} & =\int_{s_{n}}^{\infty} \widetilde{w}_{c, E^{*}}(s) d s \int_{t_{n}}^{\infty} w_{r, F}^{+}(t) d t \\
& =h_{c, E^{*}}\left(s_{n}\right) h_{r, F}\left(t_{n}\right)=\frac{s_{n} t_{n}}{g_{c, E^{*}}^{-1}\left(s_{n}\right) g_{r, F}^{-1}\left(t_{n}\right)} .
\end{aligned}
$$

However, by (3.9)

$$
\frac{1}{g_{c, E^{*}}^{-1}\left(s_{n}\right)} \sim \widetilde{w}_{c, E^{*}}\left(s_{n}\right)=\frac{1}{n}
$$

whence

$$
s_{n} \sim g_{c, E^{*}}(n) .
$$

Similar estimates hold for $g_{r, F}^{-1}\left(t_{n}\right)$. It follows that

$$
\lambda_{3} \sim g_{c, E^{*}}(n) g_{r, F}(n) .
$$

For $\lambda_{2}$ we find

$$
\frac{\lambda_{2}}{n}=\int_{0}^{s_{n}} \int_{t(s)}^{\infty} w_{r, F}^{+}(t) d t d s=\int_{0}^{s_{n}} h_{r, F}(t(s)) d s=\int_{0}^{s_{n}} \frac{t(s)}{g_{r, F}^{-1}(t(s))} d s .
$$

However,

$$
\frac{1}{g_{r, F}^{-1}(t(s))} \sim w_{r, F}^{+}(t(s))=\widetilde{w}_{c, E^{*}}(s) \sim \frac{1}{g_{c, E^{*}}^{-1}(s)}
$$

Thus

$$
t(s) \sim g_{r, F}\left(g_{c, E^{*}}^{-1}(s)\right) .
$$

Consequently,

$$
\lambda_{2} \sim n \int_{0}^{g_{c, E^{*}}(n)} \frac{g_{r, F}\left(g_{c, E^{*}}^{-1}(s)\right)}{g_{c, E^{*}}^{-1}(s)} d s
$$


The same calculation applies to $\lambda_{1}$ and yields

$$
\lambda_{1} \sim n \int_{0}^{g_{r, F}(n)} \frac{g_{c, E^{*}}\left(g_{r, F}^{-1}(t)\right)}{g_{r, F}^{-1}(t)} d t .
$$

Combining the preceding estimates we obtain

$$
\begin{aligned}
& c^{2}\|1\|_{\sqrt{n} A_{-+}+n B_{-+}}^{2} \geq g_{c, E^{*}}(n) g_{r, F}(n)+ \\
& n \int_{0}^{g_{c, E^{*}}(n)} \frac{g_{r, F}\left(g_{c, E^{*}}^{-1}(s)\right)}{g_{c, E^{*}}^{-1}(s)} d s+n \int_{0}^{g_{r, F}(n)} \frac{g_{c, E^{*}}\left(g_{r, F}^{-1}(t)\right)}{g_{r, F}^{-1}(t)} d t .
\end{aligned}
$$

To prove the converse inequality it suffices to note that the right hand side above corresponds to the decomposition $1=a+b$ with

$$
a=\mathbb{1}_{\Lambda_{1}}+\mathbb{1}_{\Lambda_{2}} \in A_{-+} \quad \text { and } \quad b=\mathbb{1}_{\Lambda_{3}} \in B_{-+} .
$$

We have

$$
\sqrt{n}\|a\|_{A_{-+}}=\sqrt{\lambda_{1}+\lambda_{2}} \text { and } n\|b\|_{B_{-+}}=\sqrt{\lambda_{3}} .
$$

Although not needed, it is helpful to observe that $b$ is a tensor, so its norm in $B_{-+}$(the projective norm) coincides with its Hilbert tensor norm. Thus

$$
\|1\|_{\sqrt{n} A_{-+}+n B_{-+}} \leq \sqrt{\lambda_{1}+\lambda_{2}}+\sqrt{\lambda_{3}} .
$$

Therefore, we deduce

$$
\begin{aligned}
& \|1\|_{\sqrt{n} A_{-+}+n B_{-+}}^{2} \sim g_{c, E^{*}}(n) g_{r, F}(n)+ \\
& n \int_{0}^{g_{c, E^{*}}(n)} \frac{g_{r, F}\left(g_{c, E^{*}}^{-1}(s)\right)}{g_{c, E^{*}}^{-1}(s)} d s+n \int_{0}^{g_{r, F}(n)} \frac{g_{c, E^{*}}\left(g_{r, F}^{-1}(t)\right)}{g_{r, F}^{-1}(t)} d t .
\end{aligned}
$$

By symmetry, we also find

$$
\begin{aligned}
& \|1\|_{\sqrt{n} A_{+-}+n B_{+-}}^{2} \sim g_{r, E^{*}}(n) g_{c, F}(n)+ \\
& n \int_{0}^{g_{r, E^{*}}(n)} \frac{g_{c, F}\left(g_{r, E^{*}}^{-1}(s)\right)}{g_{r, E^{*}}^{-1}(s)} d s+n \int_{0}^{g_{c, F}(n)} \frac{g_{r, E^{*}}\left(g_{c, F}^{-1}(t)\right)}{g_{c, F}^{-1}(t)} d t .
\end{aligned}
$$

Combining (4.7), (4.8) and (4.9), we finally get

$$
\begin{aligned}
\pi_{1}^{o}\left(\operatorname{id}_{n}\right)^{2} \sim & g_{c, E^{*}}(n) g_{r, F}(n)+g_{r, E^{*}}(n) g_{c, F}(n)+ \\
& n \int_{0}^{g_{c, E^{*}}(n)} \frac{g_{r, F}\left(g_{c, E^{*}}^{-1}(t)\right)}{g_{c, E^{*}}^{-1}(t)} d t+n \int_{0}^{g_{r, F}(n)} \frac{g_{c, E^{*}}\left(g_{r, F}^{-1}(t)\right)}{g_{r, F}^{-1}(t)} d t+ \\
& n \int_{0}^{g_{r, E^{*}}(n)} \frac{g_{c, F}\left(g_{r, E^{*}}^{-1}(t)\right)}{g_{r, E^{*}}^{-1}(t)} d t+n \int_{0}^{g_{c, F}(n)} \frac{g_{r, E^{*}}\left(g_{c, F}^{-1}(t)\right)}{g_{c, F}^{-1}(t)} d t .
\end{aligned}
$$

By the definition of the weights in consideration and (3.9) we have $g_{c, E^{*}} \sim \Phi_{c, E^{*}}$ and similar equivalences for all other functions. This allows us to replace the functions $g$ above by the respective fundamental functions. On the other hand, since all functions in consideration are equivalent to 1 on $(0,1)$, the parts on $(0,1)$ of all integrals above can be disregarded. Finally, recalling $\varphi_{n} \sim \pi_{1}^{o}\left(\mathrm{id}_{n}\right)$, we complete the proof of the theorem.

Remark 4.6 Assume that the two pairs of weights in Proposition 4.5 satisfy (3.1). Then the preceding proof shows that the infimum in (4.6) can be restricted to indicator functions $a$ and $b$. 
Theorem 0.4 clearly implies that $\pi_{1}^{o}(x) \sim \pi_{1}^{o}\left(x^{*}\right)$ for any $x \in \Pi_{1}^{o}(E, F)$ and $E, F \in H Q S(C \oplus R)$. This fact is true for general $E, F \in Q S(C \oplus R)$ :

Proposition 4.7 Let $E, F \in Q S(C \oplus R)$. Then $\pi_{1}^{o}(x) \sim \pi_{1}^{o}\left(x^{*}\right)$ for any $x \in \Pi_{1}^{o}(E, F)$ with universal equivalence constants.

Proof. Since $E$ and $F$ have the completely bounded approximation property with a universal constant, we can assume that both $E$ and $F$ are finite dimensional. On the other hand, recall that any space $Q S(C \oplus R)$ completely embeds into a noncommutative $L_{1}(\mathcal{M})$ with $\mathcal{M}$ a QWEP. It then remains to apply Lemma 4.3 .

\section{$5 \quad$ Injectivity and exactness}

Recall that an operator space $F$ is called injective if the identity map of $F$ factors through $B(H)$ by completely bounded maps for some Hilbert space $H$, or equivalently, if $F$ is completely complemented in $B(H)$. Let $F$ be a (completely isometric) subspace of $B(H)$ for some Hilbert space $H$. The projection or injectivity constant of $F$ is then defined to be

$$
\lambda_{c b}(F)=\inf \left\{\|P\|_{c b}: F \subset B(H) \text { as subspace, } P: B(H) \rightarrow F \text { projection }\right\} .
$$

Using Proposition 4.7 and the trace duality between the completely 1-summing norm and the $\gamma_{\infty}$-norm (see [J1, Lemma 4.6]), we immediately deduce the following

Proposition 5.1 Let $F \in Q S(C \oplus R)$. Then $\lambda_{c b}(F) \sim \lambda_{c b}\left(F^{*}\right)$ with universal equivalence constants.

Lemma 5.2 Let $F$ be an n-dimensional $\lambda$-homogeneous $\mu$-Hilbertian operator space. Then

$$
n \leq \pi_{1}^{o}\left(\mathrm{id}_{F}\right) \lambda_{c b}(F) \leq \lambda \mu n .
$$

Proof. This lemma is proved in [J1] for the $n$-dimensional operator Hilbert space $O H_{n}$ (see the proof of [J1, Corollary 4.11]). The proof there uses only the 1-homogeneity of $O H_{n}$; so it remains valid for general homogeneous Hilbertian operator spaces. We omit the details.

Proof of Theorem 0.3 . This is immediate from Lemma 5.2. Theorems 0.4 and 3.11.

Now we turn to the exactness. Recall that the exactness constant of an operator space $F$ is defined by

$$
e x(F)=\sup _{E \subset F, \operatorname{dim}} \inf _{E \subset \infty} d_{C b}(E, G) .
$$

$F$ is called exact if $\operatorname{ex}(F)<\infty$. We refer to [ER2], [P5] and [P2] for more information.

The exactness constant of a subspace of a noncommutative $L_{1}$ can be also expressed as a projection constant, as shown by the following result. This explains why the exactness constants of spaces in $H Q S(C \oplus R)$ can be dealt with similarly as their projection constants. Let $F$ be an operator space. Define

$$
\widetilde{\lambda}_{c b}(F)=\inf \|P\|_{c b},
$$

where the infimum runs over all von Neumann algebras $\mathcal{N}$ such that $F \subset \mathcal{N}$ as a completely isometric subspace and all completely bounded projections $P: \mathcal{N} \rightarrow F$. Note that if we require $\mathcal{N}$ to be injective in the infimum above, then we recover the injectivity constant of $F$.

Proposition 5.3 Let $F$ be a finite dimensional subspace of $L_{1}(\mathcal{M})$ for a von Neumann algebra M. Then

$$
e x(F) \leq \widetilde{\lambda}_{c b}(F) \leq \operatorname{cex}(F),
$$

where $c$ is a universal positive constant. 
Proof. The first inequality is [P5, Corollary 17.16]. This is true without the assumption that $F \subset L_{1}(\mathcal{M})$. It remains to show the second. By the operator space Grothendieck theorem of Pisier and Shlyakhtenko [PS], the inclusion map $\iota: F \hookrightarrow L_{1}(\mathcal{M})$ factors through $C \oplus R$ by completely bounded maps. More precisely, there exist $x \in C B(F, C \oplus R)$ and $y \in C B\left(C \oplus R, L_{1}(\mathcal{M})\right)$ such that $\iota=y x$ and $\|y\|_{c b}\|x\|_{c b} \leq c e x(F)$ for some universal constant $c$. Let $S=x(F) \subset C \oplus R$. Then by $[\mathrm{X}, S$ is completely isomorphic to a completely complemented subspace $G$ of a von Neumann $\mathcal{N}$ with universal constants. Since $F$ is completely isomorphic to $S$ with constant $\|y\|_{c b}\|x\|_{c b}$, we then deduce the desired inequality.

We now pass to consider spaces in $H Q S(C \oplus R)$. Let $\left(w_{c}, w_{r}\right)$ be a pair of weights on $\mathbb{R}$ satisfying (3.1). Let $F=K_{w_{c}, w_{r}}$. Recall that $F$ is the subspace of constant functions of $G_{w_{c}, w_{r}}=$ $L_{2}^{c}\left(w_{c} ; \ell_{2}\right)+L_{2}^{r}\left(w_{r} ; \ell_{2}\right)$. Let $F_{n}$ be an $n$-dimensional subspace of $F$. By homogeneity $F_{n}$ can be assumed to be the subspace of constant functions of $G_{w_{c}, w_{r}}^{n}=L_{2}^{c}\left(w_{c} ; \ell_{2}^{n}\right)+L_{2}^{r}\left(w_{r} ; \ell_{2}^{n}\right)$. Let $\iota: F_{n} \hookrightarrow G_{w_{c}, w_{r}}^{n}$ and $q: L_{2}^{c}\left(w_{c} ; \ell_{2}^{n}\right) \oplus L_{2}^{r}\left(w_{r} ; \ell_{2}^{n}\right) \rightarrow G_{w_{c}, w_{r}}^{n}$ be the natural inclusion and quotient maps, respectively.

Lemma 5.4 With the notations above we have

$$
\operatorname{ex}\left(F_{n}\right) \leq \inf \left\{\|x\|_{c b}: x: F_{n} \rightarrow L_{2}^{c}\left(w_{c} ; \ell_{2}^{n}\right) \oplus L_{2}^{r}\left(w_{r} ; \ell_{2}^{n}\right) \text { s.t. } q x=\iota\right\} \leq c \operatorname{ex}\left(F_{n}\right) .
$$

Proof. Let $x: F_{n} \rightarrow L_{2}^{c}\left(w_{c} ; \ell_{2}^{n}\right) \oplus L_{2}^{r}\left(w_{r} ; \ell_{2}^{n}\right)$ be such that $q x=\iota$. Then $F_{n}$ is completely isomorphic to $x\left(F_{n}\right)$ with constant $\|x\|_{c b}$. Since $L_{2}^{c}\left(w_{c} ; \ell_{2}^{n}\right) \oplus L_{2}^{r}\left(w_{r} ; \ell_{2}^{n}\right)$ is exact with constant 1 , we deduce $e x\left(F_{n}\right) \leq\|x\|_{c b}$. The nontrivial part is the upper estimate. The proof of this is similar to that of Proposition [5.3] By [X], $G_{w_{c}, w_{r}}^{n}$ is completely isomorphic to a complemented subspace of a noncommutative $L_{1}$-space with universal constants. Thus using again Pisier and Shlyakhtenko's theorem, we see that the inclusion $\iota: F_{n} \hookrightarrow G_{w_{c}, w_{r}}^{n}$ factors through $C \oplus R$ by completely bounded maps. Namely, there exist $y: F_{n} \rightarrow C \oplus R$ and $z: C \oplus R \rightarrow G_{w_{c}, w_{r}}^{n}$ such that $z y=\iota$ and $\|y\|_{c b}\|z\|_{c b} \leq \lambda \operatorname{ex}\left(F_{n}\right)$. Passing to duals, we get a factorization $\iota^{*}=y^{*} z^{*}$ of $\iota^{*}$ through $R \oplus C$. Since $R \oplus C$ is injective with constant $1, z^{*}$ admits an extension $\widetilde{z}: L_{2}^{r}\left(w_{c}^{-1} ; \ell_{2}^{n}\right) \oplus L_{2}^{c}\left(w_{r}^{-1} ; \ell_{2}^{n}\right) \rightarrow R \oplus C$ with $\|\widetilde{z}\|_{c b}=\|z\|_{c b}$. Thus we have the following commutative diagram

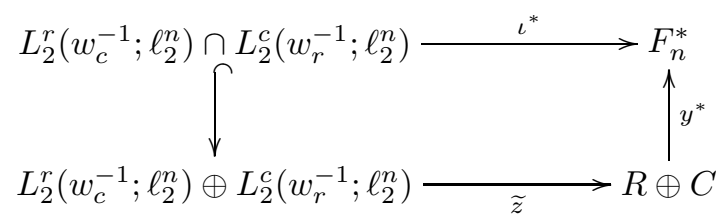

Dualizing this diagram and setting $x=\widetilde{z}^{*} y$, we get $q x=\iota$ and $\|x\|_{c b} \leq \lambda e x\left(F_{n}\right)$. This implies the desired upper estimate.

Recall that $L_{2}^{c}\left(w_{c} ; \ell_{2}^{n}\right)=\left(L_{2}\left(w_{c}\right) \otimes_{2} \ell_{2}^{n}\right)^{c}$ and $F_{n}$ is identified with $\ell_{2}^{n}$ as Banach spaces. If $a \in L_{2}\left(w_{c}\right)$, we use $a \otimes$ id to denote the map $f \mapsto a \otimes f$ from $F_{n}$ to $L_{2}^{c}\left(w_{c} ; \ell_{2}^{n}\right)$.

Lemma 5.5 Let $x: F_{n} \rightarrow L_{2}^{c}\left(w_{c} ; \ell_{2}^{n}\right) \oplus L_{2}^{r}\left(w_{r} ; \ell_{2}^{n}\right)$ be such that $q x=\iota$. Then there exist $a \in L_{2}\left(w_{c}\right)$ and $b \in L_{2}\left(w_{r}\right)$ such that $a+b=1$ a.e. and such that

$$
q \widetilde{x}=\iota \quad \text { and } \quad\|\widetilde{x}\|_{c b} \leq c\|x\|_{c b},
$$

where $\widetilde{x}: F_{n} \rightarrow L_{2}^{c}\left(w_{c} ; \ell_{2}^{n}\right) \oplus L_{2}^{r}\left(w_{r} ; \ell_{2}^{n}\right)$ is defined by $\widetilde{x}=(a \otimes \mathrm{id}, b \otimes \mathrm{id})$.

Proof. Let $\mathcal{U}$ be the unitary group of $\ell_{2}^{n}$, equipped with Haar measure. For any $u \in \mathcal{U}$ we consider $u$ as maps on both $F_{n}$ and $L_{2}^{c}\left(w_{c} ; \ell_{2}^{n}\right)$. More precisely, viewed as a map on $L_{2}^{c}\left(w_{c} ; \ell_{2}^{n}\right)=$ $\left(L_{2}^{c}\left(w_{c}\right) \otimes_{2} \ell_{2}^{n}\right)^{c}, u$ acts only on the factor $\ell_{2}^{n}$, so agrees with id $\otimes u$. Writing $x=(y, z)$, we define $\widetilde{y}: F_{n} \rightarrow L_{2}^{c}\left(w_{c} ; \ell_{2}^{n}\right)$ by

$$
\widetilde{y}=\int_{\mathcal{U}} u^{*} y u d u \text {. }
$$


More precisely,

$$
\widetilde{y}(f)=\int_{\mathcal{U}}\left(\mathrm{id} \otimes u^{*}\right)(y(u(f))) d u, \quad \forall f \in F_{n} .
$$

Similarly, we define $\widetilde{z}: F_{n} \rightarrow L_{2}^{r}\left(w_{r} ; \ell_{2}^{n}\right)$ associated to $z$. Let $\widetilde{x}=(\widetilde{y}, \widetilde{z})$. By homogeneity, $\|\widetilde{y}\|_{c b} \leq c\|y\|_{c b}$ and $\|\widetilde{z}\|_{c b} \leq c\|z\|_{c b}$. On the other hand, both $\widetilde{y}$ and $\widetilde{z}$ commute with all unitaries, so they must be multiples of $\operatorname{id}_{\ell_{2}^{n}}$. Therefore, there exist $a \in L_{2}\left(w_{c}\right)$ and $b \in L_{2}\left(w_{r}\right)$ such that

$$
\widetilde{y}=a \otimes \mathrm{id} \quad \text { and } \quad \widetilde{z}=b \otimes \mathrm{id} .
$$

Recall that $F_{n}$ is the subspace of constant functions of $L_{2}^{c}\left(w_{c} ; \ell_{2}^{n}\right)+L_{2}^{c}\left(w_{c} ; \ell_{2}^{n}\right)$, which is just $\ell_{2}^{n}$ at the algebraic level. Thus the inclusion map $\iota$ commutes with all $u \in \mathcal{U}$. On the other hand, it is clear that the quotient map $q$ also commutes with every $u$. Since $q x=\iota$, we then deduce $q \widetilde{x}=\iota$. This amounts to saying that $a+b=1$ a.e. and concludes the proof.

We are now ready to prove Theorem 0.2 ,

Proof of Theorem 0.2 We keep the notations in the proof of Theorem 0.4 and write $F=K_{w_{c}}, w_{r}$ with the pair $\left(w_{c}, w_{r}\right)$ associated to the fundamental functions of $F$. By Lemmas 5.4 and 5.5. we find

$$
e x\left(F_{n}\right) \sim \inf \left\{\|x\|_{c b}: x=(a \otimes \mathrm{id}, b \otimes \mathrm{id}), a \in L_{2}\left(w_{c}\right), b \in L_{2}\left(w_{r}\right), a+b=1 \text { a.e. }\right\} .
$$

Fix an $x=(a \otimes \mathrm{id}, b \otimes \mathrm{id})$ as above. Then

$$
\|x\|_{c b} \sim\|a \otimes \operatorname{id}\|_{C B\left(F_{n}, L_{2}^{c}\left(w_{c} ; \ell_{2}^{n}\right)\right)}+\|b \otimes \operatorname{id}\|_{C B\left(F_{n}, L_{2}^{r}\left(w_{r} ; \ell_{2}^{n}\right)\right)} .
$$

It is easy to determine the two norms on the right hand side. Indeed, we have

$$
\begin{aligned}
C B\left(F_{n}, L_{2}^{c}\left(w_{c} ; \ell_{2}^{n}\right)\right) & =L_{2}^{c}\left(w_{c} ; \ell_{2}^{n}\right) \otimes_{\min } F_{n}^{*} \\
& =L_{2}^{c}\left(w_{c}\right) \otimes_{\min } C^{n} \otimes_{\min } F_{n}^{*}=L_{2}^{c}\left(w_{c}\right) \otimes_{\min } C^{n}\left[F_{n}^{*}\right] .
\end{aligned}
$$

It then follows that

$$
\|a \otimes \mathrm{id}\|_{C B\left(F_{n}, L_{2}^{c}\left(w_{c} ; \ell_{2}^{n}\right)\right)}=\Phi_{c, F^{*}}(n)^{1 / 2}\|a\|_{L_{2}\left(w_{c}\right)} .
$$

Similarly,

$$
\|b \otimes \mathrm{id}\|_{C B\left(F_{n}, L_{2}^{r}\left(w_{r} ; \ell_{2}^{n}\right)\right)}=\Phi_{r, F^{*}}(n)^{1 / 2}\|b\|_{L_{2}\left(w_{r}\right)} .
$$

Therefore, we deduce

$$
\begin{aligned}
\operatorname{ex}\left(F_{n}\right) & \sim \inf \left\{\Phi_{c, F^{*}}(n)^{1 / 2}\|a\|_{L_{2}\left(w_{c}\right)}+\Phi_{r, F^{*}}(n)^{1 / 2}\|b\|_{L_{2}\left(w_{r}\right)}: a+b=1 \text { a.e. }\right\} \\
& \sim\|1\|_{\Phi_{c, F^{*}}(n)^{1 / 2} L_{2}\left(w_{c}\right)+\Phi_{r, F^{*}}(n)^{1 / 2} L_{2}\left(w_{r}\right)} \\
& \sim\|1\|_{L_{2}\left(\min \left(\Phi_{c, F^{*}}(n) w_{c}, \Phi_{r, F^{*}}(n) w_{r}\right)\right)}
\end{aligned}
$$

Now we apply the arguments from the proof of Theorem 0.4, where we also had to calculate the $L_{2}$-norm of 1 with respect to the minimum of two weights. We outline the main ingredients and leave the details to the reader. Indeed, on $\mathbb{R}_{+}$we have $w_{c}=1$ and hence

$$
\min \left(\Phi_{c, F^{*}}(n) w_{c}, \Phi_{r, F^{*}}(n) w_{r}\right)=\min \left(\Phi_{c, F^{*}}(n), \Phi_{r, F^{*}}(n) w_{r}\right) .
$$

The breaking point is given by $s_{n} \in \mathbb{R}_{+}$such that

$$
w_{r}\left(s_{n}\right)=\frac{\Phi_{c, F^{*}}(n)}{\Phi_{r, F^{*}}(n)} .
$$

Then

$$
s_{n} \sim \Phi_{r, F}\left(\frac{\Phi_{r, F^{*}}(n)}{\Phi_{c, F^{*}}(n)}\right)
$$


and by (3.3) and (3.9)

$$
\begin{aligned}
\|1\|_{L_{2}\left(\mathbb{R}_{+}, \min \left(\Phi_{c, F^{*}}(n) w_{c}, \Phi_{r, F^{*}}(n) w_{r}\right)\right)}^{2} & =\int_{0}^{s_{n}} \Phi_{c, F^{*}}(n) d s+\int_{s_{n}}^{\infty} \Phi_{r, F^{*}}(n) w_{r}(s) d s \\
& \sim s_{n} \Phi_{c, F^{*}}(n) \sim \Phi_{r, F}\left(\frac{\Phi_{r, F^{*}}(n)}{\Phi_{c, F^{*}}(n)}\right) \Phi_{c, F^{*}}(n) .
\end{aligned}
$$

Similarly, we have

$$
\|1\|_{L_{2}\left(\mathbb{R}_{-}, \min \left(\Phi_{c, F^{*}}(n) w_{c}, \Phi_{r, F^{*}}(n) w_{r}\right)\right)}^{2} \sim \Phi_{r, F^{*}}(n) \Phi_{c, F}\left(\frac{\Phi_{c, F^{*}}(n)}{\Phi_{r, F^{*}}(n)}\right) .
$$

Combining the preceding estimates, we obtain

$$
\operatorname{ex}\left(F_{n}\right)^{2} \sim \Phi_{c, F^{*}}(n) \Phi_{r, F}\left(\frac{\Phi_{r, F^{*}}(n)}{\Phi_{c, F^{*}}(n)}\right)+\Phi_{r, F^{*}}(n) \Phi_{c, F}\left(\frac{\Phi_{c, F^{*}}(n)}{\Phi_{r, F^{*}}(n)}\right) .
$$

Together with Theorem 3.11 , this proves the theorem.

\section{Examples}

We now apply the results in the previous sections to the column and row $p$-spaces. It is known that $C_{p} \in Q S(C \oplus R)$ for every $1<p<\infty$ (see [J1] and [X]), so $C_{p}$ can be represented as $K_{w_{c}}, w_{r}$ for a pair of weights on $\mathbb{R}$ in view of Corollary 2.7. In fact, it is easy to calculate the fundamental functions of $C_{p}$. Thus Theorem 3.8 allows us to find a concrete representation of $C_{p}$, i.e., to know explicitly the two weights $w_{c}$ and $w_{r}$. Note that such concrete representations for $C_{p}$ are not new and can be obtained by real interpolation (see $[\mathrm{X}]$ ). We should also point out that a concrete representation of $\mathrm{OH}$ was first constructed in [J1] by using Pusz-Woronowic formula on the square root of a positive sesquilinear form on a Hilbert space.

We will be also interested in the sum and intersection of $C_{p}$ and $R_{p}$. Let

$$
C R_{p}=C_{p}+R_{p} \text { for } 1 \leq p<2 \text { and } C R_{p}=C_{p} \cap R_{p} \text { for } 2 \leq p \leq \infty .
$$

These spaces are still homogenous and Hilbertian, so belong to $H Q S(C \oplus R)$ too. Their importance stems from their links to noncommutative Khintchine inequalities (see [P4]).

We start by calculating the fundamental functions of $C_{p}$ and $C R_{p}$. The following result is entirely elementary (see also $[\mathrm{X}$, Lemma 5.9]).

Proposition 6.1 Let $1 \leq p \leq \infty$ and $p^{\prime}$ be the conjugate index of $p$. Then

$$
\Phi_{c, C_{p}}(n)=n^{1 / p^{\prime}}, \quad \Phi_{r, C_{p}}(n)=n^{1 / p} \quad \text { and } \quad \Phi_{c, C R_{p}}(n)=\Phi_{r, C R_{p}}(n)=n^{1 / p^{\prime}} .
$$

Proof. We have

$$
C\left[C_{p}\right]=(C[C], C[R])_{1 / p}=\left(S_{2}, \mathbb{K}\left(\ell_{2}\right)\right)_{1 / p}=S_{2 p^{\prime}} .
$$

This implies $\Phi_{c, C_{p}}(n)=n^{1 / p^{\prime}}$. The second formula for $C_{p}$ is obtained in the same way. On the other hand, for $1 \leq p<2$ we have

$$
C\left[C R_{p}\right]=C\left[C_{p}\right]+C\left[R_{p}\right]=S_{2 p^{\prime}}+S_{2 p}=S_{2 p^{\prime}} ;
$$

whence $\Phi_{c, C R_{p}}(n)=n^{1 / p^{\prime}}$. The remaining formulas are proved similarly.

The result above shows that $C_{p}$ and $C R_{p}$ are regular for $1<p<\infty$. Thus all results in the previous sections apply to these spaces. In particular, we can determine the completely 1summing maps between them, their injectivity and exactness constants. We collect all these in the 
following three statements. In the sequel $\psi$ will denote the Orlicz function defined by $\psi(0)=0$ and $\psi(t)=t^{2} \log (t+1 / t)$ for $t>0$. It is clear that $\psi$ satisfies the $\Delta_{2}$-condition. The associated Orlicz space $\ell_{\psi}$ is traditionally denoted by $\ell^{2} \log \ell$. Note that the inverse function of $\psi$ satisfies

$$
\psi^{-1}(t) \sim \sqrt{2 t}\left(\log \frac{1}{t}\right)^{-1 / 2} \quad \text { as } \quad t \rightarrow 0 .
$$

It follows that the fundamental sequence $\left(\psi_{n}\right)$ of $\psi$ is given by

$$
\psi_{n} \sim \sqrt{n \log (n+1)} \text { as } n \rightarrow \infty .
$$

Theorem 6.2 Let $1<p, q<\infty$.

(i) Let $r$ be determined by $2 / r=1 / p+1 / q$. Then

$$
\Pi_{1}^{o}\left(C_{p}, C_{p^{\prime}}\right)=S_{\psi} \quad \text { and } \quad \Pi_{1}^{o}\left(C_{p}, C_{q}\right)=S_{\min \left(r, r^{\prime}\right)} \text { for } q \neq p^{\prime} .
$$

(ii) Let $s$ be determined by $2 / s=1 / p+1 / q^{\prime}$. Then

$$
\Pi_{1}^{o}\left(C R_{p}, C R_{p}\right)=S_{\psi} \quad \text { and } \quad \Pi_{1}^{o}\left(C R_{p}, C R_{q}\right)=S_{\min (2, s)} \text { for } q \neq p .
$$

Moreover, all relevant constants depend only on $p$ and $q$.

Proof. We consider only the part concerning $C_{p}$, the one on $C R_{p}$ being dealt with similarly. By Theorem 4.2, we know that $\Pi_{1}^{o}\left(C_{p}, C_{q}\right)=S_{\varphi}$ for some Orlicz function $\varphi$. Since $S_{\varphi}$ is completely determined by the fundamental sequence of $\varphi$ (see Corollary 4.4), we are reduced to determine $\pi_{1}^{o}\left(\mathrm{id}_{n}: C_{p}^{n} \rightarrow C_{q}^{n}\right)$ for all $n$. This is just simple integral calculations with help of Theorem 0.4 and Proposition 6.1. Indeed, we have

$$
\begin{aligned}
& \pi_{1}^{o}\left(\operatorname{id}_{n}: C_{p}^{n} \rightarrow C_{q}^{n}\right)^{2} \sim n^{1 / p+1 / q}+n^{1 / p^{\prime}+1 / q^{\prime}}+ \\
& \quad n\left[\int_{1}^{n^{1 / p}} t^{-p / q^{\prime}} d t+\int_{1}^{n^{1 / p^{\prime}}} t^{-p^{\prime} / q} d t+\int_{1}^{n^{1 / q^{\prime}}} t^{-q^{\prime} / p} d t+\int_{1}^{n^{1 / q}} t^{-q / p^{\prime}} d t\right] .
\end{aligned}
$$

If $q=p^{\prime}$, then we deduce

$$
\pi_{1}^{o}\left(\operatorname{id}_{n}: C_{p}^{n} \rightarrow C_{q}^{n}\right) \sim \sqrt{n}+\sqrt{n \log (n+1)} \sim \sqrt{n \log (n+1)} .
$$

Since $(\sqrt{n \log (n+1)})_{n \geq 1}$ is the fundamental sequence of $\psi$, we get $\Pi_{1}^{o}\left(C_{p}, C_{p^{\prime}}\right)=S_{\psi}$.

Assume $q>p^{\prime}$. Then

$$
\begin{aligned}
& \pi_{1}^{o}\left(\operatorname{id}_{n}: C_{p}^{n} \rightarrow C_{q}^{n}\right) \sim n^{1 / r}+n^{1 / r^{\prime}}+ \\
& n^{1 / 2}\left[\int_{1}^{n^{1 / p^{\prime}}} t^{-p^{\prime} / q} d t+\int_{1}^{n^{1 / q^{\prime}}} t^{-q^{\prime} / p} d t\right]^{1 / 2} \sim n^{1 / \min \left(r, r^{\prime}\right)} .
\end{aligned}
$$

Again we are done. The case $q<p^{\prime}$ is treated by symmetry on $p^{\prime}$ and $q$.

Remark 6.3 Tracking back the origin of the equivalence constants in Theorem 6.2, one can find an explicit estimate for them in terms of $p$ and $q$ and then one realizes that the result for $q=p^{\prime}$ may be obtained from that for $q \neq p^{\prime}$ by a limit procedure as $q \rightarrow p^{\prime}$.

Remark 6.4 It is easy to prove the inclusions $\Pi_{1}^{o}\left(C_{p}, C_{q}\right) \subset S_{\min \left(r, r^{\prime}\right)}$ for $q \neq p^{\prime}$ and $\Pi_{1}^{o}\left(C R_{p}, C R_{q}\right)=$ $S_{\min (2, s)}$ for $q \neq p$ are contractive. Let us show the first one. 
Let $u \in \Pi_{1}^{o}\left(C_{p}, C_{q}\right)$. Let $v: C \rightarrow C_{p}$ and $w: C_{q} \rightarrow C$ be two finite rank maps. By the ideal property of completely 1-summing norms, we have $\pi_{1}^{o}(w u v) \leq\|w\|_{c b} \pi_{1}^{o}(u)\|v\|_{c b}$. However, $C B\left(C, C_{p}\right)=S_{2 p}$ and $C B\left(C_{q}, C\right)=S_{2 q}$. It then follows that

$$
\|w u v\|_{1} \leq\|w\|_{2 q} \pi_{1}^{o}(u)\|v\|_{2 p} .
$$

Taking the supremum over all $v$ and $w$ in the unit balls of $S_{2 p}$ and $S_{2 q}$, respectively, we obtain

$$
\|u\|_{r^{\prime}} \leq \pi_{1}^{o}(u)
$$

whence

$$
\Pi_{1}^{o}\left(C_{p}, C_{q}\right) \subset S_{r^{\prime}} .
$$

Note that the argument above remains valid with column spaces replaced by row spaces. Thus $\Pi_{1}^{o}\left(R_{p}, R_{q}\right) \subset S_{r^{\prime}}$ contractively. However, $C_{p}=R_{p^{\prime}}$ and $C_{q}=R_{q^{\prime}}$. It then follows that

$$
\Pi_{1}^{o}\left(C_{p}, C_{q}\right)=\Pi_{1}^{o}\left(R_{p^{\prime}}, R_{q^{\prime}}\right) \subset S_{r} .
$$

Therefore, we deduce $\Pi_{1}^{o}\left(C_{p}, C_{q}\right) \subset S_{r} \cap S_{r^{\prime}}=S_{\min \left(r, r^{\prime}\right)}$ as desired.

The following gives the injectivity constants of $C_{p}^{n}$ and $C R_{p}^{n}$ for $p \neq 2$. Recall that $C_{2}^{n} \cong$ $C R_{2}^{n} \cong O H^{n}$ completely isometrically. Combining [J1] and [PS] we find

$$
\lambda_{c b}\left(C_{2}^{n}\right)=\lambda_{c b}\left(C R_{2}^{n}\right)=\lambda_{c b}\left(O H^{n}\right) \sim \frac{\sqrt{n}}{\sqrt{\log (n+1)}} .
$$

Theorem 6.5 Let $1<p<\infty$ such that $p \neq 2$. Then

$$
\lambda_{c b}\left(C_{p}^{n}\right) \sim n^{\frac{1}{\max \left(p, p^{\prime}\right)}} \quad \text { and } \quad \lambda_{c b}\left(C R_{p}^{n}\right) \sim \frac{\sqrt{n}}{\sqrt{\log (n+1)}}
$$

with equivalence constants depending only on $p$.

Proof. This is an immediate consequence of Theorem 6.2 and Lemma 5.2 Alternately, we can directly apply Theorem 0.3 .

The second formula in the following is already in [J3] (see Proposition 3.3.1.5 and Corollary 3.3.1.16 there), the first has been known only for $p \in\{1,2, \infty\}$.

Theorem 6.6 Let $1<p<\infty$. Then

$$
\operatorname{ex}\left(C_{p}^{n}\right) \sim n^{\frac{1}{p p^{\prime}}} \quad \text { and } \quad \operatorname{ex}\left(C R_{p}^{n}\right) \sim n^{\frac{1}{2 p}}
$$

with equivalence constants depending only on $p$.

Proof. This follows immediately from Theorem 0.2 and Proposition 6.1,

Remark 6.7 It is clear that Theorem 6.6 holds for $p=1$ and $p=\infty$. It is also obvious that the first estimate in Theorem 6.5 remains true for these endpoints. On the other hand, one has $\lambda_{c b}\left(C R_{1}^{n}\right)=\lambda_{c b}\left(C R_{\infty}^{n}\right)=\sqrt{n}$. Regarding Theorem 6.2 one can check, without difficulty, that both second equivalences in (i) and (ii) there hold if one of $p$ and $q$ is 1 or $\infty$.

Acknowledgements. This project got started in BIRS at Banff in May/June 2004 while the authors were carrying out a research team program there. They would like to thank BIRS for providing excellent research facilities. They are also very grateful to the anonymous referee for a careful reading of the manuscript and for many helpful suggestions. 


\section{References}

[B] J. Bourgain. Some remarks on Banach spaces in which martingale difference sequences are unconditional. Ark. Mat., 21:163-168, 1983.

[Bu1] D. L. Burkholder. A geometrical characterization of Banach spaces in which martingale difference sequences are unconditional. Ann. Probab., 9:997-1011, 1981.

[Bu2] D. L. Burkholder. A geometric condition that implies the existence of certain singular integrals of Banach-space-valued functions. In Conference on harmonic analysis in honor of Antoni Zygmund, Vol. I, II (Chicago, Ill., 1981), Wadsworth Math. Ser., pages 270-286. Wadsworth, Belmont, CA, 1983.

[ER1] Ed. Effros and Z.-J. Ruan. The Grothendieck-Pietsch and Dvoretzky-Rogers theorems for operator spaces. J. Funct. Anal., 122:428-450, 1994.

[ER2] Ed. Effros and Z-J. Ruan. Operator spaces. The Clarendon Press Oxford University Press, New York, 2000.

[GK] I. C. Gohberg and M. G. Kreĭn. Introduction to the theory of linear nonselfadjoint operators. American Mathematical Society, Providence, R.I., 1969.

[HM1] U. Haagerup and M. Musat. On the best constants in noncommutative Khintchine-type inequalities. J. Funct. Anal., 250:588-624, 2007.

[HM2] U. Haagerup and M. Musat. The EffrosRuan conjecture for bilinear forms on $\mathrm{C}^{*}$-algebras. Invent. Math., 174:139-163, 2008.

[J1] M. Junge. Embedding of the operator space $O H$ and the logarithmic 'little Grothendieck inequality'. Invent. Math., 161:225-286, 2005.

[J2] M. Junge. Operator spaces and Araki-Woods factors: a quantum probabilistic approach. IMRP Int. Math. Res. Pap., pages Art. ID 76978, 87, 2006.

[J3] M. Junge. Factorization theory for Spaces of Operators. Habilitationsschrift, Kiel 1996.

[K1] E. Kirchberg. On nonsemisplit extensions, tensor products and exactness of group $C^{*}-$ algebras. Invent. Math., 112:449-489, 1993.

[K2] E. Kirchberg. Exact $\mathrm{C}^{*}$-algebras, tensor products, and the classification of purely infinite algebras. In Proceedings of the International Congress of Mathematicians, Vol. 1, 2 (Zürich, 1994), pages 943-954, Basel, 1995. Birkhäuser.

[K3] E. Kirchberg. On subalgebras of the CAR-algebra. J. Funct. Anal., 129:35-63, 1995.

[LT] J. Lindenstrauss and L. Tzafriri. Classical Banach spaces. I. Springer-Verlag, Berlin, 1977.

[OR] T. Oikhberg and É. Ricard. Operator spaces with few completely bounded maps. Math. Ann., 328:229-259, 2004.

[P1] G. Pisier. Factorization of linear operators and geometry of Banach spaces, volume 60 of CBMS Regional Conference Series in Mathematics. Published for the Conference Board of the Mathematical Sciences, Washington, DC, 1986.

[P2] G. Pisier. Exact operator spaces. Astérisque, 232:159-186, 1995. Recent advances in operator algebras (Orléans, 1992).

[P3] G. Pisier. The operator Hilbert space OH, complex interpolation and tensor norms. Mem. Amer. Math. Soc., 122(585):viii+103, 1996. 
[P4] G. Pisier. Non-commutative vector valued $L_{p}$-spaces and completely $p$-summing maps. Astérisque, (247):vi+131, 1998.

[P5] G. Pisier. Introduction to operator space theory. Cambridge University Press, Cambridge, 2003.

[P6] G. Pisier. Completely bounded maps into certain Hilbertian operator spaces. Int. Math. Res. Not., (74):3983-4018, 2004.

[PS] G. Pisier and D. Shlyakhtenko. Grothendieck's theorem for operator spaces. Invent. Math., 150:185-217, 2002.

[S] B. Simon. Trace ideals and their applications. Cambridge University Press, Cambridge, 1979 .

[X] Q. Xu. Embedding of $C_{q}$ and $R_{q}$ into noncommutative $L_{p}$-spaces, $1 \leq p<q \leq 2$. Math. Ann., 335:109-131, 2006.

[Y] K-L Yew. Completely p-summing maps on the operator Hilbert space OH. J. Funct. Anal., 255:1362-1402, 2008.

M.J.: Department of Mathematics, University of Illinois, Urbana, IL 61801, USA

junge@math.uiuc.edu

Q.X.: Laboratoire de Mathématiques, Université de Franche-Comté, 25030 Besançon Cedex, France qxu@univ-fcomte.fr 